\title{
ON THE GROUPS OF C-PROJECTIVE TRANSFORMATIONS OF COMPLETE KÄHLER MANIFOLDS
}

\author{
VLADIMIR S. MATVEEV AND KATHARINA NEUSSER
}

\begin{abstract}
We show that for any complete connected Kähler manifold, the index of the group of complex affine transformations in the group of c-projective transformations is at most two unless the Kähler manifold is isometric to complex projective space equipped with a positive constant multiple of the Fubini-Study metric. This establishes a stronger version of the recently proved Yano-Obata conjecture for complete Kähler manifolds.
\end{abstract}

\section{INTRODUCTION}

Suppose $(M, J)$ is a complex manifold of real dimension $2 n \geq 4$ and $g$ a Kähler metric on $(M, J)$ with Levi-Civita connection $\nabla$. A regular curve $\gamma: I \rightarrow M$ defined on some interval $I \subseteq \mathbb{R}$ is said to be $J$-planar with respect to $\nabla$ (or $g$ ), if there exist functions $\alpha, \beta: I \rightarrow \mathbb{R}$ such that

$$
\nabla_{\gamma^{\prime}(t)} \gamma^{\prime}(t)=\alpha \gamma^{\prime}(t)+\beta J\left(\gamma^{\prime}(t)\right) \text { for all } t \in I .
$$

It follows from the definition that the property of being $J$-planar for a curve is independent of the parameterisation of the curve and that geodesics of $\nabla$ are $J$-planar curves. The $J$ planar curves form however a much larger family of curves than the family of geodesics - at every point and in every direction there exist infinitely many geometrically different $J$-planar curves. Two Kähler metrics on $(M, J)$ are called c-projectively equivalent, if they have the same $J$-planar curves, and a $c$-projective transformation of a Kähler manifold $(M, J, g)$ is a complex diffeomorphism of $M$ mapping $J$-planar curves to $J$-planar curves.

C-projective equivalence of Kähler metrics was first introduced in [OT] and provided a prominent research direction in the Japanese and Soviet schools of differential geometry, see e.g. [Mik, [S, Y]. Later, it was rediscovered under different names and with different motivations. In particular, c-projectively equivalent metrics on a given Kähler manifold are essentially the same as Hamiltonian 2-forms [ACG, CMR, and in dimension $\geq 6$ there are also essentially the same as conformal Killing (or twistor) (1,1)-forms (see [ACG, App. A] or also [MR, §1.3]), and they are closely related to the so-called Kähler-Liouville integrable systems of type $A$, see e.g. $\mathrm{KT}$. For an overview of the current developments and the renewed interest in c-projective geometry see also [CEMN.

Given a Kähler manifold $(M, J, g)$ we shall write

$$
\operatorname{Iso}(J, g) \subseteq \operatorname{Aff}(J, g) \subseteq \operatorname{CProj}(J, g)
$$

for the groups of complex isometries, of complex affine transformations (complex diffeomorphisms of $M$ preserving the Levi-Civita connection) and of c-projective transformations respectively.

2010 Mathematics Subject Classification. 32Q15, 32J27, 53A20, 53C24, 22F50, 37J35.

The paper was started during the visit of K.N. to Jena supported by DAAD Ostpartnerschaft-Programm and DFG GRK 1523. K.N. was also supported by GACR P201/12/G028. 
For the Fubini-Study metric $g_{F S}$ on complex projective space $\mathbb{C P}^{n}$ it is well-known that $\operatorname{Iso}\left(J, g_{F S}\right)=\operatorname{Aff}\left(J, g_{F S}\right)$ and also that $\operatorname{Aff}\left(J, g_{F S}\right)$ is a proper subgroup of $\operatorname{CProj}\left(J, g_{F S}\right)$. Indeed, the $J$-planar curves on $\mathbb{C P}^{n}$ (with respect to $g_{F S}$ ) are precisely those smooth regular curves that lie within complex lines (see e.g. [MR, Ex.1]) and hence $\operatorname{CProj}\left(J, g_{F S}\right)$ can be identified with the complex projective linear group $\operatorname{PGL}(n+1, \mathbb{C}) \cong \operatorname{PSL}(n+1, \mathbb{C})$. Note that an element in $\mathrm{GL}(n+1, \mathbb{C})$ induces a complex isometry of $g_{F S}$ if and only if it is proportional to a unitary isomorphism of $\mathbb{C}^{n+1}$, which shows that $\operatorname{Isom}\left(J, g_{F S}\right)$ can be identified with the Lie group $\mathrm{PU}(n+1)=\mathrm{U}(n+1) / \mathrm{U}(1)$ of projective unitary transformations. Hence, $\operatorname{CProj}\left(J, g_{F S}\right) / \operatorname{Aff}\left(J, g_{F S}\right)$ has infinitely many elements. In CEMN it was however recently shown that $g_{F S}$ (up to multiplications by positive constants and isometries) is the only complete Kähler metric for which $\operatorname{Aff}_{0}\left(J, g_{F S}\right)$ is a proper subgroup of $\operatorname{CProj}_{0}\left(J, g_{F S}\right)$, where the subscript 0 denotes the connected components of the identity of the groups. This has answered affirmatively the so-called Yano-Obata conjecture for complete Kähler manifoldsa metric c-projective analogue of the projective and conformal Lichnerowicz conjectures (see [M1, M2, KM, BMR] respectively [Fer1, Fer2, Ob, Sch, F]):

Theorem 1.1. CEMN, Theorem 7.6] Let $(M, g, J)$ be a complete connected Kähler manifold of real dimension $2 n \geq 4$. Then, $\operatorname{Aff}_{0}(J, g)=\operatorname{CProj}_{0}(J, g)$ unless $(M, g, J)$ has constant positive holomorphic sectional curvature.

In the compact case Theorem 1.1 was first proved (using different methods and crucially compactness) in [FKMR, $\mathrm{MR}$ ] and also generalised to the pseudo-Kähler setting in [BMR].

Hence, by Theorem 1.1, there are no flows of non-affine c-projective transformations on a connected complete Kähler manifold unless it is isometric to $\left(\mathbb{C P}^{n}, J, c g_{F S}\right)$ for some positive constant $c \in \mathbb{R}$. There are nevertheless examples of complete Kähler metrics other than positive constant multiples of the Fubini-Study metric for which $\operatorname{Aff}(J, g)$ is still a proper subgroup of $\operatorname{CProj}(J, g)$. These examples can be constructed using a similar idea as in [M2, $\S 1.3]$ and in these examples the index of $\operatorname{Aff}(J, g)$ in $\operatorname{CProj}(J, g)$ is two. The aim of this paper is to show the following theorem:

Theorem 1.2. Suppose $(M, J, g)$ is a connected complete Kähler manifold of real dimension $2 n \geq 4$ whose holomorphic sectional curvature is not a positive constant. Then, the index of the subgroup $\operatorname{Aff}(J, g)$ in the group $\operatorname{CProj}(J, g)$ is at most 2.

As we will explain in Section 5, as a consequence we will also obtain:

Theorem 1.3. Suppose $(M, J, g)$ is a connected complete Kähler manifold of real dimension $2 n \geq 4$ whose holomorphic sectional curvature is not a nonnegative constant. If $\operatorname{Aff}(J, g) \subsetneq$ $\operatorname{CProj}(J, g)$, then the following statements hold:

- $\operatorname{Isom}(J, g)=\operatorname{Aff}(J, g)$

- $\operatorname{Isom}(J, g)$ has index 2 in $\operatorname{CProj}(J, g)$.

Let us also remark that the group of complex affine transformations of a complete connected Kähler manifold $(M, J, g)$ is well understood: the universal cover of $(M, J, g)$ decomposes according to the de Rham decomposition into a product of Kähler manifolds

$$
\left(M_{0}, J_{0}, g_{0}\right) \times\left(M_{1}, J_{1}, g_{1}\right) \times \ldots \times\left(M_{k}, J_{k}, g_{k}\right),
$$

where $\left(M_{0}, J_{0}, g_{0}\right)$ is complex Euclidean space and $\left(M_{i}, J_{i}, g_{i}\right)$ for $1 \leq i \leq k$ a complete simply-connected Kähler manifold with irreducible holonomy. Any complex affine transformation preserves the flat factor $\left(M_{0}, J_{0}, g_{0}\right)$ and acts as a complex affine transformation on it, and permutes the factors $M_{i}$ for $1 \leq i \leq k$. Moreover, for $i=1, \ldots, k$ one has that $\left.\phi\right|_{M_{i}}$ 
is an isometry if $\phi\left(M_{i}\right)=M_{i}$, and a homothety (or an isometry) onto its image otherwise, see for example $[\mathrm{L}$, Chapter IV].

1.1. Structure of the proof of Theorem 1.2 and relation to previous results. An important role in the proof of Theorem 1.2 will be played by the metrisability equation (4) on a Kähler manifold, which is a c-projectively invariant linear overdetermined system of PDEs of finite type; we shall recall its definition and key properties in Section 2.1. The set of c-projectively equivalent metrics on a Kähler manifold $(M, J, g)$ embeds as an open subset into the vector space $\mathcal{S}$ of its solutions and the dimension of $\mathcal{S}$ is called the degree of mobility of $(M, J, g)$.

Since for a complete Kähler metric $g$ and $\phi \in \operatorname{CProj}(J, g)$, the pull-back $\phi^{*} g$ is a cprojectively equivalent complete Kähler metric, CEMN, Corollary 7.3] implies:

Theorem 1.4. Suppose $(M, J, g)$ is a connected complete Kähler manifold of real dimension $2 n \geq 4$ whose holomorphic sectional curvature is not a positive constant. If the degree of mobility of $(M, J, g)$ is at least 3 , then $\operatorname{Aff}(J, g)=\operatorname{CProj}(J, g)$.

Note moreover that on a Kähler manifold $(M, J, g)$ of degree of mobility 1 any c-projectively equivalent metric is necessarily a nonzero constant multiple of $g$. Hence, in this case any c-projective transformation is necessarily a homothety and one has $\operatorname{Aff}(J, g)=\operatorname{CProj}(J, g)$. Thus, in view of Theorem 1.4, it remains to prove Theorem 1.2 under the assumption of degree of mobility 2.

Suppose now $(M, J, g)$ satisfies the assumptions of Theorem 1.2 and is of degree of mobility 2. Since the metrisability equation is c-projectively invariant, the group of c-projective transformations of $g$ naturally acts on its solution space defining a representation of $\operatorname{CProj}(J, g)$ on the 2-dimensional vector space $\mathcal{S}$. In Section 4 , based on a circle of ideas also used in [CEMN, Z], we will analyse this representation. In Proposition 4.1 we show that, if $\phi \in \operatorname{CProj}(J, g) \backslash \operatorname{Aff}(J, g)$ acts on $\mathcal{S}$ as an isomorphism with positive determinant, then this isomorphism is necessarily diagonalisable with two distinct positive eigenvalues. Our investigations in Sections 3 and 4.2 then show that this however can never be the case, that is, $\phi \in \operatorname{CProj}(J, g) \backslash \operatorname{Aff}(J, g)$ necessarily acts on $\mathcal{S}$ as an isomorphism with negative determinant. This in turn implies that the index of $\operatorname{Aff}(J, g)$ in $\operatorname{CProj}(J, g)$ is at most 2 (see Proposition 4.3), which hence establishes Theorem 1.2.

Let us give some additional comments on the proof of Theorem 1.2 respectively Proposition 4.3 and its relation to previous results. Zeghib showed in [Z, Theorem1.3] that for compact Riemannian manifolds $(M, g)$ other than finite quotients of the standard sphere $(\operatorname{dim}(M) \geq 2)$ the group of affine transformations $\operatorname{Aff}(g)$ has at most index $2 \operatorname{dim}(M)$ in the group $\operatorname{Proj}(g)$ of projective transformations (diffeomorphisms of $M$ sending geodesics to geodesics). The first author improved this result in [M3] showing that the index is in fact at most two, and also generalised it in M4 to complete Riemannian manifolds. Let us remark that Zeghib also claimed in $[\mathbb{Z}, \S 1.2]$ - without proof though, that one can show analogously to the proof of his projective result [Z, Theorem 1.3] that on compact Kähler manifolds other than complex projective space equipped with (a positive constant multiple of) the Fubini-Study metric the index of $\operatorname{Aff}(J, g)$ in $\operatorname{CProj}(J, g)$ is finite.

The ideas of [Z, M3, M4] are also used in the proof of our Theorem 1.2, Let us emphasise that the proof of our Theorem 1.2 is however not a straightforward application of the methods of [CEMN] and the c-projective analogues of the techniques in [Z, M3, M4]. To establish Proposition 4.3 we first proceed similar as in the proof of the Yano-Obata conjecture in [CEMN, Theorem 7.6], which allows to reduce our considerations to a very special 
case (see Lemma 4.4). To handle this special case however - a step not needed in the projective case (see [M4, Z]), new arguments are needed, which we develop in Sections 3 and 4.2 .

\section{Acknowledgements}

We would like to thank the referee for helpful comments and suggestions leading to improvements of our article.

\section{C-Projective structures}

In this section we recall some background on c-projective structures and review some properties of the geodesic flows of Kähler manifolds that admit c-projectively equivalent Kähler metrics; for details we refer to [CEMN] and the references therein.

2.1. Notations. Suppose $(M, J)$ is a complex manifold of real dimension $2 n \geq 4$. When it is convenient, we will use standard abstract index notation for tensors on $(M, J)$. To avoid any confusion with the notation in [CEMN, let us emphasise that in [CEMN] the Greek alphabet is used to index real tensors on $(M, J)$, whereas the Roman alphabet is used to index complex tensors. Since we will in this paper only work inside the real setting, we will use Roman indices for tensors following the usual conventions for tensors on manifolds.

We call a linear connection $\nabla$ on $T M$ complex, if $\nabla J=0$. Two complex linear connections $\widehat{\nabla}$ and $\nabla$ on $T M$ are called c-projectively-equivalent, if they have the same $J$-planar curves (note that (11) is well-defined for any connection). If both connections are torsion-free this is known to be equivalent [OT] to the existence of a 1-form $\Upsilon_{a} \in \Gamma\left(T^{*} M\right)$ such that

$$
\widehat{\nabla}_{a} X^{b}=\nabla_{a} X^{b}+\frac{1}{2}\left(\Upsilon_{a} X^{b}+\delta_{a}{ }^{b} \Upsilon_{c} X^{c}-J_{a}{ }^{c} \Upsilon_{c} J_{d}{ }^{b} X^{d}-J_{a}{ }^{b} \Upsilon_{c} J_{d}{ }^{c} X^{d}\right) .
$$

Definition 2.1. A c-projective structure on $(M, J)$ is an equivalence class $[\nabla]$ of c-projectively equivalent torsion-free complex linear connections on $T M$. A c-projective transformation of a c-projective manifold $(M, J,[\nabla])$ is a complex diffeomorphism of $M$ preserving $[\nabla]$, equivalently mapping $J$-planar curves to $J$-planar curves.

Recall that on a complex manifold the real line bundle $\Lambda^{2 n} T^{*} M$ is canonically oriented and hence one can form an $(n+1)$ st positive root of this bundle:

$$
L:=\left(\Lambda^{2 n} T^{*} M\right)^{\frac{1}{n+1}} \quad \text { such that } L^{\otimes^{n+1}}=\Lambda^{2 n} T^{*} M .
$$

Specifically, if $\left\{\left(U_{\alpha}, u_{\alpha}\right)\right\}_{\alpha \in I}$ is an oriented atlas of $M$, then the cocycle of transition functions $U_{\alpha} \cap U_{\beta} \rightarrow \mathrm{GL}(1, \mathbb{R})$ defining $L$ is given by

$$
x \mapsto \operatorname{det}\left(T_{u_{\beta}(x)}\left(u_{\alpha} \circ u_{\beta}^{-1}\right)\right)^{-\frac{1}{n+1}} .
$$

Since the line bundles $\Lambda^{2 n} T^{*} M$ and $L$ are canonically oriented, they are trivialisable by the choice of a positive section, but there is no canonical trivialisation of these bundles on a complex or c-projective manifold. In the sequel we also use for $L$ or $L^{*}$-valued tensors abstract index notation, e.g. $X^{a}$ may denote simply a vector field or a section of $T M \otimes L$ respectively $T M \otimes L^{*}$; what is meant will be always clear from the context. Let us also

remark that for two connections $\widehat{\nabla}$ and $\nabla$ on $T M$ related as in (2), their induced connections on $L$ are related by

$$
\widehat{\nabla}_{a} \sigma=\nabla_{a} \sigma-\Upsilon_{a} \sigma
$$


Moreover, we will denote by $S_{+}^{2} T^{*} M$ the bundle of $J$-Hermitian symmetric covariant tensors on $(M, J)$, that is, $h_{a b} \in \Gamma\left(S_{+}^{2} T^{*} M\right)$, if

$$
h_{a b}=h_{(a b)} \quad \text { and } \quad J_{a}^{c} J_{b}{ }^{d} h_{c d}=h_{a b} .
$$

Note that, by definition, any (pseudo-)Kähler metric $g_{a b}$ on $(M, J)$ is a non-degenerate section of $S_{+}^{2} T^{*} M$ and as usual we denote its inverse by $g^{a b} \in \Gamma\left(S_{+}^{2} T M\right)$, which is characterised by $g_{a b} g^{b c}=\delta_{a}^{c}$ (in index-free notation we also write $g^{-1}$ for the inverse of $g$ ). We denote by $\operatorname{vol}(g) \in \Gamma\left(\Lambda^{2 n} T^{*} M\right)$ the volume form of $g$. Given a (pseudo-)Kähler metric $g$ on $(M, J)$, one can form the $(n+1)$ st root of its volume form $\operatorname{vol}(g)$ (viewed as a positive section of an oriented line bundle), which naturally defines a positive section $\operatorname{vol}(g)^{\frac{1}{n+1}}$ of $L$. Hence, on a (pseudo-)Kähler manifold $\operatorname{vol}(g)$ respectively $\operatorname{vol}(g)^{\frac{1}{n+1}}$ canonically trivialise $\Lambda^{2 n} T^{*} M$ respectively $L$ and hence sections of both these line bundles can be canonically identified with functions. Note also that any (pseudo-)Kähler metric gives rise to a c-projective structure via its Levi-Civita connection.

2.2. Metrisability equation. Suppose $(M, J,[\nabla])$ is a c-projective manifold of real dimension $2 n \geq 4$. Then the Leibniz rule together with (2) and (3) implies that the trace-free part of $\nabla_{a} \eta^{b c}$ for any section $\eta^{b c} \in \Gamma\left(S_{+}^{2} T M \otimes L\right)$ is independent of the choice of connection $\nabla \in[\nabla]$. Hence, in this sense the so-called metrisability equation, given by

$$
\nabla_{a} \eta^{b c}-\delta_{a}{ }^{(b} X^{c)}-J_{a}^{(b} J_{d}^{c)} X^{d}=0,
$$

where $X^{a}=\frac{1}{n} \nabla_{a} \eta^{a b} \in \Gamma(T M \otimes L)$, is c-projectively invariant. A detailed analysis of the geometry and algebra of this equation can be found in [CEMN], we recall here only the properties relevant for this article:

- Since (4) is linear, its solution space $\mathcal{S}$ is a real vector space.

- C-projective invariance implies that the pull-back of any solution of (4) by a cprojective transformation is again a solution.

- If $(M, J,[\nabla])$ admits a compatible Kähler metric, that is $[\nabla]$ contains the Levi-Civita connection of a Kähler metric $g$ of $(M, J)$, then the section

$$
\eta^{a b}:=g^{a b} \operatorname{vol}(g)^{\frac{1}{n+1}}:=g^{a b} \otimes \operatorname{vol}(g)^{\frac{1}{n+1}} \in \Gamma\left(S_{+}^{2} T M \otimes L\right)
$$

defines a solution of (4), since $g$ and consequently $\operatorname{vol}(g)^{\frac{1}{n+1}}$ are parallel for the LeviCivita connection of $g$. In fact, by [CEMN, Proposition 4.5], mapping an Hermitian metric $g_{a b}$ (of arbitrary signature) on $(M, J)$ to $\eta^{a b}$ defined by (5) restricts to a bijection between compatible (pseudo-)Kähler metrics of $(M, J,[\nabla])$ and solutions of (4) that are non-degenerate (viewed as bundle maps $T^{*} M \times T^{*} M \rightarrow L$ ) at any point of $M$.

- Note also that, since $L$ is an oriented line bundle, we have not only a well defined notion for sections $T^{*} M \times T^{*} M \rightarrow L$ of $S_{+}^{2} T M \otimes L$, in particular of $\mathcal{S}$, to be nondegenerate at a point but also to be positive-definite, negative-definite and indefinite at a point, since these notions are independent of the choice of a positive trivialising section of $L$.

Remark 2.2. Since (4) is an overdetermined system of PDEs, for a generic c-projective structure, one has $\mathcal{S}=\{0\}$. In particular, a c-projective structure has generically no compatible (pseudo-)Kähler metrics. 
Suppose now $(M, J, g)$ is a (pseudo-)Kähler manifold with Levi-Civita connection $\nabla$ and consider the induced c-projective structure $(M, J,[\nabla])$. Then we can use $g$ to identify contravariant with covariant tensors and $\operatorname{vol}(g)^{\frac{1}{n+1}}$ to trivialise $L$. In particular, any section $\eta^{a b} \in \Gamma\left(S_{+}^{2} T M \otimes L\right)$ can be identified with a $(J, g)$-Hermitian endomorphism $A$ of $T M$ given by

$$
A_{a}{ }^{b}:=\operatorname{vol}(g)^{-\frac{1}{n+1}} \eta^{b c} g_{c a} \in \Gamma(\operatorname{End}(T M)),
$$

where $\operatorname{vol}(g)^{-\frac{1}{n+1}} \in \Gamma\left(L^{*}\right)$ is the dual section of $\operatorname{vol}(g)^{\frac{1}{n+1}} \in \Gamma(L)$ (note that $L \otimes L^{*}$ is the trivial bundle $M \times \mathbb{R})$. Moreover, solutions of (4) can be identified with $(J, g)$-Hermitian endomorphism $A \in \Gamma(\operatorname{End}(T M))$ that satisfy (with respect to the Levi-Civita connection $\nabla$ of $g$ )

$$
\nabla_{a} A_{b}{ }^{c}=\frac{1}{2}\left(g_{a b} \Lambda^{c}+\delta_{a}^{c} \Lambda_{b}+\Omega_{a b} J_{d}^{c} \Lambda^{d}+J_{a}^{c} \Lambda^{d} \Omega_{d b}\right) \quad \text { for some } \Lambda^{c} \in \Gamma(T M),
$$

where $\Omega_{a b}:=J_{a}{ }^{c} g_{c b}$ is the Kähler form of $g$ and $\Lambda_{a}:=\Lambda^{b} g_{b a}$. Note that, if we set $\lambda:=\frac{1}{2} A_{a}{ }^{a}$, then $\Lambda^{a}$ equals the gradient $\operatorname{gr}(\lambda)$ of $\lambda$ with respect to $g$. The equation (77) is precisely the form of the metrisability equation that was used in [DM, FKMR, $[$ ] to study c-projectively equivalent Kähler metrics; we will refer to it also as the mobility equation.

In summary, $g$ induces an isomorphism of vector spaces

$$
\mathcal{S} \simeq \operatorname{Sol}(J, g),
$$

where

$$
\operatorname{Sol}(J, g):=\left\{A_{a}{ }^{b} \in \Gamma(\operatorname{End}(T M)): A \text { is }(J, g) \text {-Hermitian and satisfies (77) }\right\} .
$$

Note that invertible elements in $\operatorname{Sol}(J, g)$ correspond to (everywhere) non-degenerate elements in $\mathcal{S}$ and hence to (pseudo-)Kähler metrics that are c-projectively equivalent to $g$. Given an invertible solution $A \in \operatorname{Sol}(J, g)$ the corresponding c-projectively equivalent metric $\tilde{g}$ is given by

$$
\tilde{g}_{a b}=\sqrt{\operatorname{det}_{\mathbb{R}}(A)} g_{a c} B_{b}^{c}=\left|\operatorname{det}_{\mathbb{C}}(A)\right| g_{a c} B_{b}{ }^{c},
$$

where $B=A^{-1}$ and $\operatorname{det}_{\mathbb{R}}(A)$ and $\operatorname{det}_{\mathbb{C}}(A)$ denote the real and complex determinant of $A$, respectively (note that, since $A$ is $(g, J)$-Hermitian, $\operatorname{det}_{\mathbb{C}}(A)$ is a real-valued function). Moreover, $\tilde{g}$ is evidently affinely equivalent to $g$ (i.e. $\nabla$ is also the Levi-Civita connection of $\tilde{g})$ if and only if $A$ is $\nabla$-parallel.

Since $\operatorname{Sol}(J, g)$ always contains the identity, one can at least locally add to any solution $A \in \operatorname{Sol}(J, g)$ always an appropriate multiple of the identity to obtain an invertible element of $\operatorname{Sol}(J, g)$. Hence, locally the dimension of $\operatorname{Sol}(J, g)$ coincides with the number of linearly independent compatible (pseudo-)Kähler metrics of $(M, J,[\nabla])$. Further let us remark that the equation (4) (respectively (7D) is of finite type and prolongation shows that its solutions are in bijection to parallel sections of a linear connection on a vector bundle of rank $(n+1)^{2}$ (see [CEMN, DM]). Hence, the vector space $\mathcal{S}$ (respectively $\operatorname{Sol}(J, g)$ ) is of dimension at most $(n+1)^{2}$. As already mentioned in the introduction we call the dimension of $\mathcal{S}$ (respectively $\operatorname{Sol}(J, g))$ the degree of mobility of $(M, J,[\nabla])$ (respectively $(M, J, g)$ ).

For later purpose we also recall the following fact:

Proposition 2.3. Suppose $(M, J, g)$ is a (pseudo-)Kähler manifold of dimension $2 n \geq 4$. Then for any solution $A \in \operatorname{Sol}(J, g)$ of the mobility equation the corresponding vector field $\Lambda$ is holomorphic (i.e. its (local) flowpreserves $J$ ) and $K:=J \Lambda$ is even a holomorphic Killing vector field with respect to $(J, g)$, which is equivalent to $\nabla \Lambda$ being $(J, g)$-Hermitian. Moreover, $\nabla \Lambda$ commutes with $A$. 
Proof. The first statement is well known in c-projective geometry; see e.g. [CEMN, Proposition 5.6], [FKMR, Lemma 1] or in the language of Hamiltonian 2-forms [ACG, Proposition 3]. A proof of the second statement can be found in [DM], CEMN, Proposition 5.13] or also [BMR, Lemma 2.2(7)].

2.3. Metric c-projective structures and integrals for the geodesic flow. Recall that a smooth function $I: T M \rightarrow \mathbb{R}$ on a (pseudo-)Riemannian manifold $(M, g)$ is called an integral of the geodesic flow (or an integral) of $g$, if for any affinely parametrised geodesic $\gamma$ the function $s \mapsto I\left(\gamma^{\prime}(s)\right)$ is constant.

Suppose now $(M, J, g)$ is a (pseudo-)Kähler manifold of dimension $2 n \geq 4$. In Proposition 2.3 we have already noted that a solution $A \in \operatorname{Sol}(J, g)$ of the mobility equation (7) gives rise to a holomorphic Killing vector field $K$ and hence to a linear integral for the geodesic flow of $g$. In fact, it is known that all coefficients of the characteristic polynomial of $A$ are generators of holomorphic Killing vector fields and hence give rise to linear integrals of $g$ (see [ACG, Proposition 3], [CEMN, Theorem 5.11(1)]); we will however only need Proposition 2.3 in this article.

An essential tool in our article will be that any solution $A \in \operatorname{Sol}(J, g)$ of the mobility equation gives also rise to a family of quadratic integrals $I_{t}$ of $g$ as shown by Topalov in Top. For $t \in \mathbb{R}$ the integral $I_{t}$ is given by

$$
I_{t}: T M \rightarrow \mathbb{R}, \quad I_{t}(X)=g\left(\operatorname{det}_{\mathbb{C}}(A(t)) A(t)^{-1} X, X\right),
$$

where $A(t):=t \mathrm{Id}-A$ and $\operatorname{det}_{\mathbb{C}}(A(t))$ denotes the complex determinant of $A(t)$ (which is here viewed as a complex $n \times n$-matrix). Note that $\operatorname{det}_{\mathbb{C}}(A(t)) A(t)^{-1}$ is a polynomial of degree $n-1$ in $t$, whose coefficients give rise to integrals of $g$. Moreover, it was shown in [CEMN, Theorem 5.18] that on an open dense set the degree of the minimal polynomial of $A$ (i.e. in case $g$ is positive definite, the number of different eigenvalues of $A$ ) is constant and equals the number of functionally independent integrals in the family $I_{t}$.

2.4. C-projective Weyl curvature. Suppose $(M, J)$ is a complex manifold of real dimension $2 n \geq 4$. Let $\nabla$ be a complex torsion-free connection $\nabla$ on $T M$ and write

$$
R_{a b}{ }^{c} X^{d}:=\nabla_{a} \nabla_{b} X^{c}-\nabla_{b} \nabla_{a} X^{c} .
$$

for its curvature and $\operatorname{Ric}_{a b}:=R_{c a}{ }^{c} b$ for its Ricci tensor. Then one may decompose $R_{a b}{ }^{c} d$ as

$$
R_{a b}{ }^{c} d=W_{a b}{ }^{c}+(\partial \mathrm{P})_{a b}{ }^{c} d
$$

where

$$
\begin{aligned}
(\partial \mathrm{P})_{a b}{ }^{c} d & :=\delta_{[a}{ }^{c} \mathrm{P}_{b] d}-J_{[a}{ }^{c} \mathrm{P}_{b] e} J_{d}{ }^{e}-\mathrm{P}_{[a b]} \delta_{d}^{c}-J_{[a}{ }^{e} \mathrm{P}_{b] e} J_{d}{ }^{c} . \\
\mathrm{P}_{a b} & :=\frac{1}{n+1}\left(\operatorname{Ric}_{a b}+\frac{1}{n-1}\left(\operatorname{Ric}_{(a b)}-J_{(a}{ }^{c} J_{b)}{ }^{d} \operatorname{Ric}_{c d}\right)\right) .
\end{aligned}
$$

It can be shown (see [OT, CEMN]) that $W_{a b}{ }^{c}{ }_{d}$ does not depend on the connection in the induced c-projective class $[\nabla]$ of $\nabla$. Hence, it is an invariant of the c-projective manifold $(M, J,[\nabla])$, called its c-projective Weyl curvature.

Recall also that a Kähler metric $g$ on $(M, J)$ is said to have constant holomorphic sectional curvature $\mu \in \mathbb{R}$, if its curvature (i.e. the curvature of its Levi-Civita connection) takes the form

$$
R_{a b c d}=\frac{\mu}{4}\left(g_{a c} g_{b d}-g_{b c} g_{a d}+\Omega_{a c} \Omega_{b d}-\Omega_{b c} \Omega_{a d}+2 \Omega_{a b} \Omega_{c d}\right),
$$

where $R_{a b c d}:=R_{a b}{ }_{d} g_{e c}$ and $\Omega_{a b}:=J_{a}{ }^{c} g_{c b} \in \Gamma\left(\Lambda^{2} T^{*} M\right)$ is the Kähler-form. 
In the following theorem we collect some results which we will need in the sequel:

Theorem 2.4. Suppose $(M, J, g)$ is a Kähler manifold of dimension $2 n \geq 4$ with Levi-Civita connection $\nabla$. Then one has:

(1) $W$ vanishes identically if and only if $(M, J,[\nabla])$ is locally c-projectively flat, that is, locally c-projectively equivalent to $\left(\mathbb{C P}^{n}, J,\left[\nabla^{g_{F S}}\right]\right)$, where $\nabla^{g_{F S}}$ denotes the LeviCivita connection of the Fubini-Study metric $g_{F S}$.

(2) If $M$ is connected, then $W$ vanishes identically if and only if $(M, J, g)$ has constant holomorphic sectional curvature.

(3) If $(M, J, g)$ is connected, complete and has positive constant holomorphic sectional curvature, then $(M, J, g)$ is simply-connected and isometric to $\left(\mathbb{C P}^{n}, J, c g_{F S}\right)$ for some positive constant $c$.

Proof. Statement (3) is a standard fact in Kähler geometry and for (1) and (2) see [T] or CEMN, Theorems 2.16 and 4.2].

Remark 2.5. For a general c-projective manifold (which is not necessarily induced by a Kähler metric) statement (1) of Theorem 2.4 still holds provided $2 n \geq 6$. If $2 n=4$, the c-projective Weyl curvature is in general not sufficient to characterise c-projective flatness. It turns out that in this case the vanishing of $W$ and a part of the c-projective Cotton-York tensor is what characterises c-projectively flat structures, see [CEMN, Theorem 2.16].

Remark 2.6. We already mentioned that on a Kähler manifold $(M, J, g)$ of real dimension $2 n \geq 4$ solutions of the mobility equation are in bijection to parallel sections of a linear connection on a vector bundle of rank $(n+1)^{2}$, see e.g. [CEMN, Theorem. 4.16]. Hence, if $M$ is simply-connected, $\operatorname{dim}(\operatorname{Sol}(J, g))=(n+1)^{2}$ if and only if this connection has vanishing curvature, which in turn can be shown to be the case if and only if the c-projective Weyl curvature vanishes. In particular, in view of Theorem 2.4 , on $\left(\mathbb{C P}^{n}, J, g_{F S}\right)$ we have $\operatorname{dim}\left(\left(\operatorname{Sol}\left(J, g_{F S}\right)\right)=(n+1)^{2}\right.$.

\section{KÄHLER MANIFOLDS With A VERY SPECIAL TYPE OF SOLUTION OF THE MOBILITY EQUATION}

In this section we study the topology of complete Kähler manifolds admitting a solution of the mobility equation of a very restrictive type. We show that the existence of such a solution implies that the manifold is compact. This will be a crucial ingredient in the proof of our main Theorem 1.2 .

3.1. Some general facts. Suppose $(M, J, g)$ is a connected Kähler manifold and $A \in$ $\operatorname{Sol}(J, g)$ a solution of the mobility equation. Since $g$ is assumed to be positive-definite and $A$ is $(J, g)$-Hermitian, at any point of the manifold $A$ is diagonalisable with real eigenvalues and any eigenvalue is of even (real) algebraic multiplicity.

Definition 3.1. A point $x \in M$ is called regular with respect to $A$, if

- the number of distinct eigenvalues of $A$ is constant on a neighbourhood of $x$,

- for a smooth eigenvalue $\rho$ defined on a neighbourhood of $x$ either $d \rho(x) \neq 0$ or $\rho$ is constant on a neighbourhood of $x$.

We denote the set of regular points by $M_{\text {reg. }}$. Note that $M_{\text {reg }}$ is an open and dense subset of $M$. Moreover, the following can be shown (see [CEMN, Lemma 5.16, Corollary 5.17] or also [BMR, Lemma 2.2]): 
Proposition 3.2. Suppose $(M, J, g)$ is a connected Kähler manifold of dimension $2 n \geq 4$ and $A \in \operatorname{Sol}(J, g)$ a solution of the mobility equation. Then we have:

(1) At any regular point the algebraic (real) multiplicity of any non-constant eigenvalue $\rho$ of $A$ is 2 and its eigenspace is spanned by its gradient $\operatorname{gr}(\rho)$ and its skew-gradient $\operatorname{Jgr}(\rho)$.

(2) If an eigenvalue $\rho$ of $A$ is constant around some regular point $x$ (i.e. $d \rho(x)=0$ ), then the constant $\rho$ is an eigenvalue at any point of $M$ and its (real) algebraic multiplicity is constant on the set of regular points.

3.2. Solutions of the mobility equation of special type. Let $(M, J, g)$ be a connected Kähler manifold of dimension $2 n \geq 4$ and $A \in \operatorname{Sol}(J, g)$ a solution of the mobility equation with the following property:

(P) it has locally around any regular point the following structure of eigenvalues:

- two constant eigenvalues 1 and 0 of multiplicity $2 m$ and $2 \tilde{m}$ respectively,

- one non-constant eigenvalue $\rho$ with values in $(0,1)$ of multiplicity 2 ,

where $m, \tilde{m} \in \mathbb{Z}_{\geq 0}$ are arbitrary such that $n-m-\tilde{m}=1$.

Note that assumption (P) implies that $\rho=\lambda-m$, where $\lambda=\frac{1}{2} A_{a}{ }^{a}$. Since $\lambda$ defines a smooth function on all of $M$, we can also extend $\rho$ from a smooth function defined on the set of regular points to a smooth function defined on the whole manifold $M$ by dint of this equality. We set

$$
M_{0}:=\{x \in M: \rho(x)=0\} \text { and } M_{1}:=\{x \in M: \rho(x)=1\} .
$$

Since $\rho$ has extrema at points of $M_{0}$ and $M_{1}$, we have

$$
M_{0} \subseteq N \quad \text { and } \quad M_{1} \subseteq N,
$$

where $N$ is the zero set of the gradient vector field $\Lambda:=\operatorname{gr}(\lambda)=\operatorname{gr}(\rho) \neq 0$. Since $N$ also coincides with the zero set of $J \Lambda$, which is a (holomorphic) Killing vector field by Proposition 2.3. $N$ is a union of closed connected totally geodesic submanifolds, each of which has even dimension at most $2 n-2$.

Remark 3.3. To avoid any ambiguity, let us remark that closed submanifold of $M$ here and everywhere else in this article, in particular in Propositions 3.5 and 3.9 below, means that it is a closed subset of $M$, not that it is compact.

For later purposes, note that for any point $x \in M$ we can find a basis of $T_{x} M$, which we call adapted, in which $g_{x}=\mathrm{Id}=\operatorname{diag}(1, \ldots, 1)$ and $A_{x}$ and $J_{x}$ have the following blockdiagonal form:

$$
A_{x}=\left(\begin{array}{ccc}
\rho(x) \operatorname{Id}_{2} & & \\
& \operatorname{Id}_{2 m} & \\
& & 0_{2 \tilde{m}}
\end{array}\right) \text { and } J_{x}=\left(\begin{array}{ccccc}
0 & -1 & & & \\
1 & 0 & & & \\
& & \ddots & & \\
& & 0 & -1 \\
& & 1 & 0
\end{array}\right) .
$$

For a vector $\xi \in T_{x} M$ we shall denote by $\xi_{i}$ the coordinates with respect to a chosen adapted basis of $T_{x} M$. In such coordinates, the family of quadratic integral $I_{t}$ (8) induced by $A$ has the form:

$$
I_{t}(\xi)=(t-1)^{\alpha(m)(m-1)} t^{\alpha(\tilde{m})(\tilde{m}-1)} \tilde{I}_{t}(\xi)
$$


where

$\tilde{I}_{t}(\xi)=(t-1)^{\alpha(m)} t^{\alpha(\tilde{m})}\left(\xi_{1}^{2}+\xi_{2}^{2}\right)+(t-\rho) t^{\alpha(\tilde{m})}\left(\xi_{3}^{2}+\ldots+\xi_{2 m+2}^{2}\right)+(t-\rho)(t-1)^{\alpha(m)}\left(\xi_{2 m+3}^{2}+\ldots+\xi_{2 n}^{2}\right)$, and $\alpha(\ell)$ equals 1 for $\ell \geq 1$ and 0 for $\ell=0$. Note that for fixed $t$ the coefficient $(t-1)^{\alpha(m)(m-1)} t^{\alpha(\tilde{m})(\tilde{m}-1)}$ is a constant and hence $\tilde{I}_{t}(\xi)$ also forms a family of integrals for the geodesic flow of $g$.

The goal of this section is to prove:

Theorem 3.4. Suppose $(M, J, g)$ is a connected complete Kähler manifold of dimension $2 n \geq 4$ and let $A \in \operatorname{Sol}(J, g)$ be a solution of the mobility equation that satisfies (P). If $M_{0}$ and $M_{1}$ are both not empty, then $M$ is compact.

The proof will be based on several propositions:

Proposition 3.5. Suppose $(M, J, g)$ is a connected Kähler manifold of dimension $2 n \geq 4$ and $A \in \operatorname{Sol}(J, g)$ satisfies property $(\mathrm{P})$. Then one of the following statements holds for $M_{0}$ (respectively $\left.M_{1}\right)$ :

- $M_{0}$ (resp. $\left.M_{1}\right)$ is empty, or if not,

- $M_{0}\left(\right.$ resp. $\left.M_{1}\right)$ is a discrete subset of $M$ provided $m=0$ (resp. $\left.\tilde{m}=0\right)$ and a closed totally geodesic Kähler submanifold of dimension $2 m$ (resp. $2 \tilde{m}$ ) provided $m \geq 1$ (resp. $\tilde{m} \geq 1$ ) whose tangent space at any point is the eigenspace of $A$ with eigenvalue 1 (resp. 0). In particular, if $M$ is complete, then $M_{0}$ (resp. $M_{1}$ ) is complete.

Proof. It suffices to prove the statement for $M_{0}$, since replacing $A$ by $\operatorname{Id}-A$ (which obviously also satisfies (P)) interchanges $M_{0}$ and $M_{1}$.

Suppose $M_{0} \neq \emptyset$. Fix a point $x_{0} \in M_{0}$ and a convex neighbourhood $U$ of $x_{0}$ and let $\varepsilon>0$ be sufficiently small such that the image of

$$
S_{x_{0}}^{\varepsilon}:=\left\{\xi \in T_{x_{0}} M: \xi_{i}=0 \text { for } i=1,2 \text { or } i \geq 2 m+3, \text { and } \xi_{3}^{2}+\ldots+\xi_{2 m+2}^{2}<\varepsilon\right\}
$$

under the exponential map is contained in $U$. We aim to show that, by possibly shrinking $U$, we can achieve that $\exp \left(S_{x_{0}}^{\varepsilon}\right)=U \cap M_{0}$. Note that, since $x_{0} \in M_{0}$ was arbitrary, this would imply that $M_{0}$ is a totally geodesic closed Kähler submanifold of dimension $2 m$ (or a discrete subset, if $m=0$ ) as desired.

Now consider the family $\tilde{I}_{t}$ of integrals defined as in (12) and let $\gamma:[0,1] \rightarrow U$ be a geodesic with $\gamma(0)=x_{0}$ and $\gamma^{\prime}(0)=\left(\xi_{1}, \ldots, \xi_{2 n}\right) \in S_{x_{0}}^{\varepsilon} \backslash\{0\}$. Then,

$$
\tilde{I}_{t}\left(\gamma^{\prime}(0)\right)=t^{\alpha(\tilde{m})+1}\left(\xi_{3}^{2}+\ldots+\xi_{2 m+2}^{2}\right),
$$

which has a zero of order $\alpha(\tilde{m})+1$ at $t=0$. Hence the same must be true at $\gamma(1)$ and substituting $t=0$ in (12) we therefore obtain

$\tilde{I}_{0}\left(\gamma^{\prime}(1)\right)=(-1)^{\alpha(m)} 0^{\alpha(\tilde{m})}\left(\xi_{1}^{2}+\xi_{2}^{2}\right)-0^{\alpha(\tilde{m})} \rho\left(\xi_{3}^{2}+\ldots+\xi_{2 m+2}^{2}\right)+(-1)^{\alpha(m)+1} \rho\left(\xi_{2 m+3}^{2}+\ldots+\xi_{2 n}^{2}\right)=0$,

where the $\xi_{i}$ 's now denote the coefficients of $\gamma^{\prime}(1)$ with respect to an adapted basis of $T_{\gamma(1)} M$.

Case 1: Suppose $\tilde{m}=0$.

Then have

$$
\tilde{I}_{0}\left(\gamma^{\prime}(1)\right)=-\left(\left(\xi_{1}^{2}+\xi_{2}^{2}\right)+\rho\left(\xi_{3}^{2}+\ldots+\xi_{2 n}^{2}\right)\right)=0,
$$

which implies $\rho(\gamma(1))=0$, since $\gamma^{\prime}(1) \neq 0$. Hence, $\exp \left(S_{x_{0}}^{\varepsilon}\right) \subset U \cap M_{0}$ in this case. Moreover, $M_{0}$ is contained in the zero set of the non-trivial Killing vector field $J \Lambda$, which equals a union of connected submanifolds of dimension at most $2 n-2$. For dimensional reasons we can hence achieve by possibly shrinking $U$ that $\exp \left(S_{x_{0}}^{\varepsilon}\right)=U \cap M_{0}$. 
Case 2: Suppose $\tilde{m} \geq 1$.

If $\rho(\gamma(1)) \neq 0$, then (13) implies that at $\gamma(1)$ we have $\xi_{i}=0$ for $2 m+3 \leq i \leq 2 n$ and hence

$$
\tilde{I}_{t}\left(\gamma^{\prime}(1)\right)=\left(t\left((t-1)^{\alpha(m)}\left(\xi_{1}^{2}+\xi_{2}^{2}\right)+(t-\rho)\left(\xi_{3}^{2}+\ldots+\xi_{2 m+2}^{2}\right)\right)\right) .
$$

Since $\tilde{I}_{t}\left(\gamma^{\prime}(1)\right)$ must have a zero of order $\alpha(\tilde{m})+1=2$ at $t=0$, this implies that also $\xi_{i}=0$ at $\gamma(1)$ for $1 \leq i \leq 2 m+2$, which contradicts our assumptions. Thus, we must have $\rho(\gamma(1))=0$ and hence $\exp \left(S_{x_{0}}^{\varepsilon}\right) \subset U \cap M_{0}$.

Now take a point $y \in U \backslash\left(U \cap M_{0}\right)$ (since the set of regular point is dense in $M$, almost every point in $U$ has this property) and let $x \in M_{0} \cap U$. Then there exists a geodesic $\gamma:[0,1] \rightarrow U$ connecting $y=\gamma(0)$ with $x=\gamma(1)$. Since $\rho$ is zero at $x$, we see that the integral $\tilde{I}_{0}$ is zero along $\gamma$ and hence we deduce from (12) that $\xi_{2 m+3}=\ldots=\xi_{2 n}=0$ at $y$. Thus, $M_{0} \cap U \subset \exp \left(V_{y}\right)$, where $V_{y}$ is the $2 m+2$-dimensional subspace of $T_{y} M$ defined by the condition $\xi_{2 m+3}=\ldots=\xi_{2 n}=0$. Since $y$ was arbitrary in $U \backslash U \cap M_{0}$, we must have

$$
M_{0} \cap U \subset \bigcap_{y \in U \backslash U \cap M_{0}} \exp \left(V_{y}\right) .
$$

Since $\exp \left(V_{y}\right)$ is locally a $2 m+2$-dimensional submanifold and $2 m+2<2 n$, the intersection $\bigcap_{y \in U \backslash U \cap M_{0}} \exp \left(V_{y}\right)$ must locally be a submanifold of dimension at most $2 \mathrm{~m}$. Since we moreover have $\exp \left(S_{x_{0}}^{\varepsilon}\right) \subset U \cap M_{0}$, we conclude again that we can arrange $\exp \left(S_{x_{0}}^{\varepsilon}\right)=M_{0} \cap U$ by possible shrinking $U$.

Using the quadratic integrals (12), we next establish some key properties of certain types of geodesics of Kähler manifolds with property $(\mathrm{P})$ :

Proposition 3.6. Suppose $(M, J, g)$ is a connected Kähler manifold of dimension $2 n \geq 4$ and $A \in \operatorname{Sol}(J, g)$ satisfies property (P). Then the following holds:

(1) Let $\gamma: I \rightarrow M$ be a geodesic and suppose there exists $s_{0} \in I$ such that $\Lambda\left(\gamma\left(s_{0}\right)\right) \neq 0$ and $\gamma^{\prime}\left(s_{0}\right)$ is proportional to $\Lambda\left(\gamma\left(s_{0}\right)\right)$. Then for all $s \in I$ such that $\Lambda(\gamma(s)) \neq 0$ the velocity vector $\gamma^{\prime}(s)$ is proportional to $\Lambda(\gamma(s))$.

(2) The distribution $D$ of rank $2 m+2$ on $M \backslash M_{0}$ generated by the eigenvectors corresponding to the eigenvalues $\rho$ and 1 is totally geodesic.

(3) If $M_{1} \neq \emptyset$, then for any geodesic $\gamma: I \rightarrow M \backslash M_{0}$ with $\gamma\left(s_{0}\right) \in M_{1}$ and $\gamma^{\prime}\left(s_{0}\right) \in$ $T_{\gamma\left(s_{0}\right)}^{\perp} M_{1}$ for some $s_{0} \in I$, the velocity vector $\gamma^{\prime}(s)$ is a $\rho$-eigenvector of $A$ for all $s \in I$. Moreover, it is proportional to $\Lambda(\gamma(s))$ provided the latter is not zero.

(4) If $M_{0} \neq \emptyset$ and $M_{1} \neq \emptyset$, then any geodesic connecting a point of $M_{0}$ with a point of $M_{1}$ is orthogonal to $M_{0}$ respectively $M_{1}$ at these points.

Proof. (1) With respect to an adapted basis of $T_{\gamma\left(s_{0}\right)} M$, the family of integrals $\tilde{I}_{t}$ defined as in (12) satisfies (we again assume $\left.\gamma^{\prime}\left(s_{0}\right)=\left(\xi_{1}, \ldots, \xi_{2 n}\right)\right)$ that

$$
\tilde{I}_{t}\left(\gamma^{\prime}\left(s_{0}\right)\right)=(t-1)^{\alpha(m)} t^{\alpha(\tilde{m})}\left(\xi_{1}^{2}+\xi_{2}^{2}\right),
$$

by Proposition $3.2(1)$. Hence, $\tilde{I}_{0}\left(\gamma^{\prime}\left(s_{0}\right)\right)=0$ for $\tilde{m} \geq 1$ and $\tilde{I}_{1}\left(\gamma^{\prime}\left(s_{0}\right)\right)=0$ for $m \geq 1$. Therefore at any point $\gamma(s)$ of the geodesic we must have

$$
\begin{aligned}
\tilde{I}_{0}\left(\gamma^{\prime}(s)\right)=(-1)^{\alpha(m)+1} \rho\left(\xi_{2 m+3}^{2}+\ldots+\xi_{2 n}^{2}\right)=0 & \text { if } \tilde{m} \geq 1, \\
\tilde{I}_{1}\left(\gamma^{\prime}(s)\right)=(1-\rho)\left(\xi_{3}^{2}+\ldots+\xi_{2 m+2}^{2}\right)=0 & \text { if } m \geq 1,
\end{aligned}
$$

where $\left\{\xi_{i}\right\}$ now denotes the coordinates of $\gamma^{\prime}(s)$ with respect to an adapted basis of $T_{\gamma(s)} M$. At points where $\rho$ equals 0 or 1 , the function $\rho$ has an extremum and so $\Lambda=\operatorname{gr}(\rho)=0$ at 
these points. Hence, we conclude from (15) and (16) that $\gamma^{\prime}(s)$ is a linear combination of $\Lambda$ and $J \Lambda$ at all points $\gamma(s)$ where these vector fields are not zero. Since $J \Lambda$ is a Killing vector field and orthogonal to $\gamma^{\prime}\left(s_{0}\right)$, the same must be true for $\gamma^{\prime}(s)$ for any $s$, which shows that $\gamma^{\prime}(s)$ is proportional to $\Lambda$ at all points $\gamma(s)$ where the latter does not vanish.

(2) Note first that for $\tilde{m}=0$ the statement is trivially satisfied, since $D=\left.T M\right|_{M \backslash M_{0}}$ in this case. Assume now $\tilde{m} \geq 1$ and consider a geodesic $\gamma: I \rightarrow M \backslash M_{0}$ such that $\gamma^{\prime}\left(s_{0}\right) \in D_{\gamma\left(s_{0}\right)}$ for some $s_{0} \in I$. Then $\tilde{I}_{0}\left(\gamma^{\prime}\left(s_{0}\right)\right)=0$ and hence for all $s \in I$ we have

$$
\tilde{I}_{0}\left(\gamma^{\prime}(s)\right)=(-1)^{\alpha(m)+1} \rho\left(\xi_{2 m+3}^{2}+\ldots+\xi_{2 n}^{2}\right)=0
$$

with respect to an adapted basis of $T_{\gamma(s)} M$. Since $\rho(\gamma(s)) \neq 0$, this implies $\gamma^{\prime}(s) \in D_{\gamma(s)}$ for all $s \in I$.

(3) Consider a geodesic $\gamma: I \rightarrow M \backslash M_{0}$ with $\gamma\left(s_{0}\right) \in M_{1}$ and $\gamma^{\prime}\left(s_{0}\right) \in T_{\gamma\left(s_{0}\right)}^{\perp} M_{1}$. Note that, by Proposition 3.5, $T_{\gamma\left(s_{0}\right)}^{\perp} M_{1}=D_{\gamma\left(s_{0}\right)}$. In particular, if $m=0$, the first part of the statement holds by $(2)$. Now suppose $m \geq 1$. Then $\tilde{I}_{1}\left(\gamma^{\prime}\left(s_{0}\right)\right)=0$ and hence $\tilde{I}_{1}\left(\gamma^{\prime}(s)\right)=0$ for all $s \in I$. By (2), this implies that $\gamma^{\prime}(s)$ is a $\rho$-eigenvector at all points with $\rho(\gamma(s)) \neq 1$ and hence at all points of $\gamma(s)$ by continuity. For the second statement, recall that, by Proposition 3.2. $\gamma^{\prime}(s)$ is a linear combination of $\Lambda$ and $J \Lambda$ at all point $\gamma(s)$ where these vector fields do not vanish. Moreover, since $\rho$ has a maximum at $\gamma\left(s_{0}\right)$, the Killing vector field $J \Lambda$ vanishes at $\gamma\left(s_{0}\right)$ and hence the inner product of $J \Lambda$ with $\gamma^{\prime}(s)$ must be zero for all $s \in I$.

(4) Consider a geodesic $\gamma:[0,1] \rightarrow M$ such that $\gamma(0) \in M_{0}$ and $\gamma(1) \in M_{1}$. Since $\rho(\gamma(0))=0$, it follows from (12) that $\tilde{I}_{0}\left(\gamma^{\prime}(0)\right)=0$. Hence, also $\tilde{I}_{0}\left(\gamma^{\prime}(1)\right)=0$ and, since $\rho(\gamma(1))=1$, we conclude from (12) and Proposition 3.5 that $\gamma^{\prime}(1) \in T_{\gamma(1)}^{\perp} M_{1}$. Similarly, since $\rho(\gamma(1))=1$, formula (12) shows $\tilde{I}_{1}\left(\gamma^{\prime}(1)\right)=0$. Hence, also $\tilde{I}_{0}\left(\gamma^{\prime}(0)\right)=0$. Since $\rho(\gamma(0))=0$, this implies, by (12) and Proposition 3.5, that $\gamma^{\prime}(0) \in T_{\gamma(0)}^{\perp} M_{0}$.

Remark 3.7. Since the roles of $M_{0}$ and $M_{1}$ exchange, if one replaces $A$ by $\operatorname{Id}-A$, the analogues of the statements (2) and (3) in Proposition 3.6 also hold for $M_{1}$.

For a solution $A \in \operatorname{Sol}(J, g)$ of the mobility equation with property (P), the set of regular points $M_{\text {reg }} \subseteq M$ as defined in Definition 3.1 simply coincides with the set of points of $M$ on which $\Lambda$ does not vanish, equivalently, on which the holomorphic Killing vector field $J \Lambda$ does not vanish. We have already mentioned that $M_{\text {reg }}$ is an open dense subset in $M$. Since $M_{\text {reg }}$ is the complement of the zero set of a non-trivial Killing vector field, it is the complement of a submanifold of co-dimension at least two and as such it is connected. To study the topology of $M$ let us consider the foliation $\mathcal{F}$ on $M_{\text {reg generated by the function }}$ $\rho$, that is, its leaves are the connected components of the level sets of $\rho$. Since $\operatorname{gr}(\rho)=\Lambda$ does not vanish on $M_{\text {reg }}$, they are connected $2 n$-1-dimensional submanifolds of $M$. We write $L(x) \subset M_{\text {reg }}$ for the leaf containing $x \in M_{\text {reg }}$.

Lemma 3.8. Suppose $(M, J, g)$ is a connected Kähler manifold of dimension $2 n \geq 4$ and $A \in \operatorname{Sol}(J, g)$ has property $(\mathrm{P})$. Let $\xi \in T_{x} M$ for some $x \in M$ be a linear combination of $K=J \Lambda$ and of eigenvectors corresponding to eigenvalues 0 and 1 . Then the derivative of the function $g(\Lambda, \Lambda)=g(K, K)$ vanishes in direction of $\xi$.

Proof. At a zero $x \in M$ of $\Lambda$ the function $g(\Lambda, \Lambda)$ has a minimum and hence its derivative vanishes at $x$. Suppose now $x \in M$ is regular, that is, $\Lambda(x) \neq 0$ (in particular $\rho(x) \neq 0,1$ ). Recall that by Proposition 2.3 the endomorphism $\nabla \Lambda$ of $T M$ commutes with $A$ and $J$, and is self-adjoint with respect to $g$. Hence, it follows from Proposition 3.2 that $\nabla_{\Lambda} \Lambda$ is a 
$\rho$-eigenvector of $A$ and, since $\nabla \Lambda$ is $(J, g)$-Hermitian, moreover that it is proportional to $\Lambda$ at $x$. Self-adjointness of $\nabla \Lambda$ with respect to $g$ (i.e. $g_{a c} \nabla_{b} \Lambda^{c}$ is symmetric in $a$ and $b$ ) implies then that $g\left(\nabla_{\xi} \Lambda, \Lambda\right)=g\left(\nabla_{\Lambda} \Lambda, \xi\right)=0$ for $\xi \in \Lambda^{\perp}$.

Proposition 3.9. Suppose $(M, J, g)$ is a connected Kähler manifold of dimension $2 n \geq 4$ and $A \in \operatorname{Sol}(J, g)$ has property $(\mathrm{P})$. Then the foliation $\mathcal{F}$ on the set of regular points $M_{\text {reg }}$ has the following properties:

(1) For any $x \in M_{\mathrm{reg}}$ the tangent space of the leaf $L(x)$ at $x$ is generated by $J \Lambda$ and the eigenvectors of $A$ with eigenvalues 0 and 1 .

(2) The function $g(\Lambda, \Lambda)$ is constant on the leaves of $\mathcal{F}$.

(3) Each leaf is a closed subset of $M$.

(4) For any $x \in M_{\text {reg }}$ there exist an $\varepsilon>0$ and a neighbourhood $U(x)$ in $M_{\text {reg }}$ such that

- for any $t \in(-\varepsilon, \varepsilon)$ the flow $\phi_{t}$ of $\Lambda$ is defined on $U(x)$ and

$$
\phi_{t}(U(x) \cap L(x)) \subseteq L\left(\phi_{t}(x)\right),
$$

- for any fixed $t \in(-\varepsilon, \varepsilon)$ the distance function $y \mapsto \operatorname{dist}\left(y, \phi_{t}(y)\right)$ is constant on $U(x) \cap L(x)$.

Proof. (1) For any $x \in M_{\text {reg }}$ the tangent space $T_{x} L(x)$ is given by the kernel of $\nabla \rho$ at $x$, which equals the orthogonal complement of the gradient $\Lambda$ of $\rho$ at $x$. Hence, the statement follows from Proposition 3.2 .

(2) Since $L(x)$ is by definition connected, the statement follows from (1) and Lemma 3.8

(3) Consider a sequence $\left(x_{k}\right)_{k \in \mathbb{N}} \in L(x)$ converging to a point $\tilde{x} \in M$. Clearly, we must have $\rho(\tilde{x})=\rho(x)$, so it remains to check that $\tilde{x}$ is a regular point, i.e. $d \rho(\tilde{x}) \neq 0$. This however immediately follows from (2).

(4) Let $x \in M_{\text {reg. }}$. Then there exists $\varepsilon>0$ and a neighbourhood $U(x)$ of $x$ inside $M_{\text {reg }}$ such that $\phi_{t}$ is defined on $U(x)$ for all $t \in(-\varepsilon, \varepsilon)$. We may also arrange that $U(x) \cap L(x)$ is connected. Since $\phi_{t}$ preserves $\Lambda$ and $U(x) \cap L(x)$ is connected, $\phi_{t}(U(x) \cap L(x))$ is a connected subset contained in $M_{\text {reg }}$ and $\phi_{t}(U(x) \cap L(x)) \subseteq L\left(\phi_{t}(x)\right)$ is equivalent to $\rho \circ \phi_{t}$ being constant on $U(x) \cap L(x)$ for any fixed $t$. This in turn is equivalent, over $U(x) \cap L(x)$, to $[\Lambda, \xi] \in \Lambda^{\perp}$ for any section $\xi \in \Lambda^{\perp}$. Since $\nabla_{\xi} \Lambda \in \Lambda^{\perp}$ for $\xi \in \Lambda^{\perp}$ by Lemma 3.8 and $\nabla$ is torsion-free, the latter is equivalent to $\nabla_{\Lambda} \xi \in \Lambda^{\perp}$ for $\xi \in \Lambda^{\perp}$, which follows from differentiating $0=g(\Lambda, \xi)$ for a section $\xi \in \Lambda^{\perp}$ in direction $\Lambda$ and the fact that $\nabla_{\Lambda} \Lambda$ is proportional to $\Lambda$.

For the second property in (4), note that, by possibly shrinking $U(x)$ and $\varepsilon$, we can achieve that $U(x)$ is strictly convex and that

$$
U(x)=\left\{\phi_{t}(y): y \in U(x) \cap L(x), t \in(-\varepsilon, \varepsilon)\right\},
$$

which gives rise to flow-box coordinates on $U(x)$.

Since $\nabla_{\Lambda} \Lambda$ is proportional to $\Lambda$, for any $y \in U(x) \cap L(x)$ and $t_{0} \in(-\varepsilon, \varepsilon)$ the curve $\phi_{t}(y):\left[0, t_{0}\right] \rightarrow U(x)$ (respectively $\left[t_{0}, 0\right]$ if $t_{0}<0$ ) can be reparametrised to a geodesic segment and by strict convexity of $U(x)$ this geodesic segment is length minimising. The length of the velocity vector of the curve $\phi_{t}(y)$ is given by $\sqrt{g(\Lambda, \Lambda)}$ and by (2) it is a function of $\rho$. Thus, the length of the curve $\phi_{t}(y)$ is the same for all points $y \in U(x) \cap L(x)$ and for any fixed $t_{0} \in(-\varepsilon, \varepsilon)$ the function $y \mapsto \operatorname{dist}\left(y, \phi_{t_{0}}(y)\right)$ is constant on $U(x) \cap L(x)$.

Remark 3.10. The statements (2) and (4) of Proposition 3.9 imply the existence of a coordinate system $y_{1}, \ldots, y_{2 n}$ in a neighbourhood of every regular point such that $\rho=\rho\left(y_{1}\right)$ 
and

$$
g=d y_{1}^{2}+\sum_{i, j=2}^{2 n} h_{i j} d y_{i} d y_{j} .
$$

In this coordinate system $\Lambda=\left(\rho^{\prime}(y), 0, \ldots, 0\right)$ and its integral curves are "vertical" geodesics.

Proposition 3.11. Suppose $(M, J, g)$ is a connected complete Kähler manifold and $A \in$ $\operatorname{Sol}(J, g)$ satisfies $(\mathrm{P})$. Let $\gamma: I \rightarrow M$ be an arc length parameterised geodesic with the following properties:

(a) the domain $I$ of $\gamma$ is either a closed interval $[a, b](a, b \in \mathbb{R})$, a ray $[a,+\infty)$ or $(-\infty, b]$, or all of $\mathbb{R}$,

(b) $\Lambda(\gamma(s)):=\operatorname{gr}_{\gamma(s)}(\rho) \neq 0$ for all $s$ in the interior $I^{o}$ of $I$ and $\Lambda(\gamma(s))=0$ for all $s \in I \backslash I^{o}$,

(c) at some $s_{0} \in I^{0}$, hence by Proposition 3.6 at all $s \in I^{o}$, the velocity vector of $\gamma$ is proportional to $\Lambda$.

Then the following statements hold:

$$
M_{\mathrm{reg}}=\bigcup_{s \in I^{\circ}} L(\gamma(s)),
$$

(2) the flow $\phi_{t}$ of $\Lambda$ acts simply transitively on the set of leaves,

(3) there exist a connected $2 n-1$ dimensional manifold $L$ and a diffeomorphism

$$
\Psi: M_{\text {reg }} \rightarrow L \times I^{o}
$$

with the following properties:

- the images of the leaves of the foliation under $\Psi$ are the sets of the form $L \times\{s\}$ for $s \in I^{o}$; in particular, the push-forward of the function $\rho$ depends only on $s \in I^{\circ}$,

- the push-forward of the vector field $\Lambda$ is tangent to the lines $\{x\} \times I^{o}$, and

- for any point $x \in L$ and $s_{1} \leq s_{2} \in I^{o}$ the distance between the points $\Psi^{-1}\left(x, s_{1}\right)$ and $\Psi^{-1}\left(x, s_{2}\right)$ is $s_{2}-s_{1}$ and the shortest arc-length parameterised geodesic connecting these points is the preimage $\Psi^{-1}(\tilde{\gamma})$ of the "vertical" curve $\tilde{\gamma}:\left[s_{1}, s_{2}\right] \rightarrow$ $L \times I^{o}, s \mapsto(x, s)$.

Proof. (1) Denote by $M_{\mathrm{reg}}^{\prime} \subseteq M_{\text {reg }}$ the right-hand side of (18). Since $M_{\text {reg }}$ is connected, it is sufficient to show that $M_{\text {reg }}^{\prime}$ is open and closed in $M_{\text {reg }}$ in order to show that $M_{\text {reg }}^{\prime}=M_{\text {reg. }}$.

First, let us show that $M_{\text {reg }}^{\prime}$ is open: Fix a point $x \in L(\gamma(s))$ with $s \in I^{o}$. Since $L(x)=L(\gamma(s))$ is connected, there exists a curve $\tilde{c}:\left[0, \frac{1}{2}\right] \rightarrow L(\gamma(s))$ connecting $\tilde{c}(0)=\gamma(s)$ with $\tilde{c}\left(\frac{1}{2}\right)=x$. By (4) of Proposition 3.9 and compactness of $\left[0, \frac{1}{2}\right]$, there exists $\varepsilon>0$ such that $\tilde{c}\left(\left[0, \frac{1}{2}\right]\right)$ can be covered by neighbourhoods (in $M_{\text {reg }}$ ) of the form

$$
U(z)=\left\{\phi_{t}(z): y \in U(z) \cap L(z), t \in(-\varepsilon, \varepsilon)\right\}, \quad z \in \tilde{c}\left(\left[0, \frac{1}{2}\right]\right),
$$

satisfying the properties of (4) of Proposition 3.9. Let now $\phi_{t_{0}}(y)$ be any point in $U(x)$ (i.e. $\left|t_{0}\right|<\varepsilon$ and $\left.y \in L(x) \cap U(x)\right)$. Since $L(y)=L(x)=L(\gamma(s))$ is connected, we can extend $\tilde{c}$ to a curve $c:[0,1] \rightarrow L(\gamma(s))$ connecting $c(0)=\gamma(s)$ with $c(1)=y$. By construction, the curve $\phi_{t_{0}} \circ c$ connecting $\phi_{t_{0}}(\gamma(s))$ with $\phi_{t_{0}}(y)$ is well-defined and lies inside one leaf. By (c), $\phi_{t_{0}}(\gamma(s))$ must lie on $\gamma\left(I^{o}\right)$, which shows that $\phi_{t_{0}}(y) \in M_{\text {reg }}^{\prime}$. Hence, $M_{\text {reg }}^{\prime}$ is open in $M_{\text {reg }}$. 
In order to show that $M_{\text {reg }}^{\prime}$ is also closed in $M_{\text {reg }}$ suppose $\left(x_{k}\right)_{k \in \mathbb{N}}$ is a sequence in $M_{\text {reg }}^{\prime}$ that converges to a point $\tilde{x} \in M_{\text {reg. }}$. Denote by $\left(s_{k}\right)_{k \in \mathbb{N}} \in I^{o}$ the corresponding sequence in $I^{o}$ such that $x_{k} \in L\left(\gamma\left(s_{k}\right)\right)$. Since $g\left(\Lambda(\gamma(s)), \gamma^{\prime}(s)\right)$ is nonzero for all $s \in I^{0}$, the function $s \mapsto \rho(\gamma(s))$ is strictly monotonic on $I$. Hence, $\rho\left(\gamma\left(s_{k}\right)\right)=\rho\left(x_{k}\right)$ implies that $\left(s_{k}\right)_{k \in \mathbb{N}}$ converges and we denote its limit by $\tilde{s} \in I$. Note moreover that Proposition 3.9 (2) implies that $g\left(\Lambda\left(\gamma\left(s_{k}\right)\right), \Lambda\left(\gamma\left(s_{k}\right)\right)\right)=g\left(\Lambda\left(x_{k}\right), \Lambda\left(x_{k}\right)\right)$ for all $k \in \mathbb{N}$. Hence, $g(\Lambda(\gamma(\tilde{s})), \Lambda(\gamma(\tilde{s})))=g(\Lambda(\tilde{x}), \Lambda(\tilde{x})) \neq 0$, which implies $\tilde{s} \in I^{o}$ by the assumptions on $\gamma$. Since $\Lambda(\gamma(s))$ is proportional to $\gamma^{\prime}(s)$ at points $s \in I^{o}$, there exists a sequence $\left(t_{k}\right)_{k \in \mathbb{N}}$ converging to 0 such that $\phi_{t_{k}}\left(\gamma\left(s_{k}\right)\right)=\gamma(\tilde{s})$. Note that the sequence $\left(\tilde{x}_{k}\right)_{k \in \mathbb{N}}:=\left(\phi_{t_{k}}\left(x_{k}\right)\right)_{k \in \mathbb{N}} \in L(\gamma(\tilde{s}))$ still converges to $\tilde{x}$. Since the leaf $L(\gamma(\tilde{s}))$ is closed in $M$ by Proposition 3.9(3), we must have $\tilde{x} \in L(\gamma(\tilde{s}))$. Hence, $M_{\text {reg }}^{\prime}$ is closed in $M_{\text {reg }}$. Finally, $M_{\text {reg }}^{\prime}=M_{\text {reg }}$.

(2) Since $\gamma\left(I^{o}\right)$ lies on a flow line of $\Lambda$ and $\Lambda$ is nowhere vanishing on $\gamma\left(I^{o}\right)$, the flow $\phi_{t}$ of $\Lambda$ acts simply transitively on $\gamma\left(I^{o}\right)$ and hence on the set of leaves by (4) of Proposition 3.9 and (1).

(3) Take $s_{0} \in I^{o}$ and set $L:=L\left(\gamma\left(s_{0}\right)\right)$. For any point $x \in M_{\text {reg }}$ it follows from (1) that there exist $s \in I^{o}$ such that $x \in L(\gamma(s))$. Since the function $s \mapsto \rho(\gamma(s))$ is strictly monotonic on $I^{o}$, this $s$ is unique. By (2) there exists a unique $t$ such that $\phi_{t}(\gamma(s))=\gamma\left(s_{0}\right) \in L$ and set $\Psi(x):=\left(\phi_{t}(x), s\right) \in L \times I^{o}$. Then $\Psi: M_{\mathrm{reg}} \rightarrow L \times I^{o}$ is a diffeomorphism as claimed. Indeed, the first two properties are satisfied by construction. In order to show the last property consider the point $\Psi^{-1}\left(x, s_{2}\right)$ and the point $y$ of the leaf $L\left(\Psi^{-1}\left(x, s_{1}\right)\right)$ which is the closest point of the leaf $L\left(\Psi^{-1}\left(x, s_{1}\right)\right)$ to $\Psi^{-1}\left(x, s_{2}\right)$, which exists by completeness of $g$ and Proposition $3.9(3)$. The minimising geodesic connecting $y$ to $\Psi^{-1}\left(x, s_{2}\right)$ is orthogonal to the leaf $L\left(\Psi^{-1}\left(x, s_{1}\right)\right)$. Hence, its velocity vector is proportional to $\Lambda$ at its initial point and so, by Proposition 3.6(1), the image of that geodesic must coincide with a segment of the flow line $\phi_{t}(y)$. In particular, $y=\Psi^{-1}\left(x, s_{1}\right)$. Arguing as at the end of the proof of Proposition 3.9 (4), we obtain that the distance between $\Psi^{-1}\left(x, s_{1}\right)$ and $\Psi^{-1}\left(x, s_{2}\right)$ equals the length of $\gamma_{\mid\left[s_{1}, s_{2}\right]}$ which in turn is equal to $s_{2}-s_{1}$.

Now we are ready to give the proof of Theorem 3.4 .

Proof of Theorem 3.4. Fix a point $x_{0} \in M_{0}$. It follows from the Hopf-Rinow Theorem that there exists a point $x_{1} \in M_{1}$ such that $\operatorname{dis}\left(x_{0}, M_{1}\right)=\operatorname{dist}\left(x_{0}, x_{1}\right)$. Moreover, completeness of $(M, J, g)$ implies the existence of a minimising geodesic $\gamma:[0,1] \rightarrow M$ connecting these points, that is, $\gamma(0)=x_{0}$ and $\gamma(1)=x_{1}$. Without loss of generality we assume that there is no other point of $M_{0}$ on $\gamma(s)$ than $x_{0}$. Without loss of generality we may also assume that $\gamma$ is parameterised by arc-length, since otherwise we just multiply the metric $g$ by the appropriate constant to achieve that.

By (3-4) of Proposition 3.6 we know that at any point $s \in[0,1]$ such that $\Lambda(\gamma(s)) \neq 0$ the velocity vector $\gamma^{\prime}(s)$ is proportional to $\Lambda(\gamma(s))$. We also know that $\Lambda\left(x_{0}\right)=\Lambda\left(x_{1}\right)=0$. Let us now show that $\Lambda(\gamma(s)) \neq 0$ for all $s \in(0,1)$, which implies that $\gamma$ satisfies the assumptions of Proposition 3.11. By contradiction, assume there exists $s_{0} \in(0,1)$ such that $\Lambda$ vanishes at $y:=\gamma\left(s_{0}\right)$. Then also the Killing vector field $K:=J \Lambda$ vanishes at $y$. We already remarked that the zero set $N$ of $K$ (being the zero set of a Killing vector field) is a union of connected (totally geodesics closed) submanifolds of codimension at least 2 in $M$. By Lemma 3.8 the property of $K$ to be zero is preserved along the integral curves of the $2 m+2 \tilde{m}$-dimensional distribution generated by the direct sum of the eigenspaces of $A$ corresponding to the eigenvalues 1 and 0 . Hence, $y \in N$ lies in a connected submanifold 
$N_{s t} \subset N$ of $M$ of codimension precisely 2. Now consider the action of the flow of $K$ on the tangent space $T_{y} M$. It acts as the identity on $T_{y} N_{s t} \subset T_{y} M$ and therefore by rotations on the 2-dimensional orthogonal compliment of $T_{y} N_{s t}$ in $T_{y} M$. Hence there exists an isometry generated by the flow that sends the vector $\gamma^{\prime}\left(s_{0}\right)$ (at the point $y$ ) to the vector $-\gamma^{\prime}\left(s_{0}\right)$. This in turn implies the existence of one more point of $M_{0} \cup M_{1}$ (different from $x_{0}, x_{1}$ ) on the geodesic segment $\gamma([0,1])$ (note that $\rho$ is invariant under the flow of $K$, hence the flow can not map $x_{0}$ to $x_{1}$ and vice versa), which contradicts our assumption. Hence, $\Lambda(\gamma(s)) \neq 0$ for all $s \in(0,1)$ and $\gamma$ satisfies the assumptions of Proposition 3.11 as claimed.

Now, by Proposition 3.11, there exists a diffeomorphism $\Psi: M_{\mathrm{reg}} \simeq L\left(\gamma\left(\frac{1}{2}\right)\right) \times(0,1)$ such that the geodesics tangent to $\Lambda$ correspond to the lines $\{x\} \times(0,1)$ inside $L\left(\gamma\left(\frac{1}{2}\right)\right) \times(0,1)$. Then, any geodesic starting orthogonally from a point of $M_{0}$ reaches in distance 1 a point of $M_{1}$. Hence, the image $\exp \left(S_{x_{0}} M_{0}\right)$ of the compact set

$$
S_{x_{0}} M_{0}:=\left\{\xi \in T_{x_{0}} M \mid g(\xi, \xi)=1, \xi \in T_{x_{0}}^{\perp} M_{0}\right\}
$$

under the exponential map is contained in $M_{1}$. In fact, $\exp \left(S_{x_{0}} M_{0}\right)$ coincides with $M_{1}$ : Let $y_{1}$ be a point in $M_{1}$, then, by completeness of $M$, there exists a minimising geodesic $\tilde{\gamma}:[0,1] \rightarrow M$ connecting $\tilde{\gamma}(0)=x_{0}$ and $\tilde{\gamma}(1)=y_{1}$. By our assumptions, the length of $\tilde{\gamma}$ must equal the length of $\gamma$, which is 1 . Hence, $\tilde{\gamma}^{\prime}(0) \in S_{x_{0}} M_{0}$ by (4) of Proposition 3.6. Therefore, $\exp \left(S_{x_{0}} M_{0}\right)=M_{1}$, which in particular implies that $M_{1}$ is compact.

Now consider the set

$$
S M_{1}:=\left\{(x, \xi) \in T M \mid x \in M_{1}, g(\xi, \xi) \leq 1, \quad \xi \in T_{x}^{\perp} M_{1}\right\} .
$$

Since $M_{1}$ is compact, $S M_{1}$ is compact as well. Arguing as above using Proposition 3.11, we conclude that its image $\exp \left(S M_{1}\right)$ contains all regular points. Since the set of regular points is dense in $M$ and $S M_{1}$ is compact, we must have $\exp \left(S M_{1}\right)=M$, which implies that $M$ is compact as claimed.

\section{Proof of Theorem 1.2}

Suppose $(M, J, g)$ is a connected complete Kähler manifold of real dimension $2 n \geq 4$. As explained in Section 1.1, it remains to prove Theorem 1.2 under the assumption that the degree of mobility of $(M, J, g)$ equals 2. Recall that, by Theorem 2.4. any connected complete Kähler manifold with constant positive holomorphic sectional curvature is compact and isometric to $\left(\mathbb{C P}^{n}, J, c \mathrm{~g}_{F S}\right)$, where $c>0$ is some positive constant. Since $\left(\mathbb{C P}^{n}, J, c \mathrm{~g}_{F S}\right)$ has degree of mobility $(n+1)^{2}>2$ by Remark 2.6. we see that the assumption of degree of mobility equal to 2 implies that $(M, J, g)$ is not of constant positive holomorphic sectional curvature.

4.1. The case of degree of mobility 2. Suppose $(M, J, g)$ is a Kähler manifold of real dimension $2 n \geq 4$, denote its Levi-Civita connection by $\nabla$ and consider the induced cprojective manifold $(M, J,[\nabla])$. The c-projective invariance of the metrisability equation (41) implies that for any c-projective transformation $\phi \in \operatorname{CProj}(J, g)$ and any $\eta \in \mathcal{S}$ we have $\phi^{*} \eta \in \mathcal{S}$. Moreover, the map

$$
\begin{aligned}
& T: \operatorname{CProj}(J, g) \times \mathcal{S} \rightarrow \mathcal{S} \\
&(\phi, \eta) \mapsto\left(\phi^{-1}\right)^{*} \eta
\end{aligned}
$$

evidentially defines a representation of the group $\operatorname{CProj}(J, g)$ on the (finite-dimensional) vector space $\mathcal{S}$. We set $T_{\phi}:=T(\phi,-) \in \operatorname{GL}(\mathcal{S}, \mathbb{R})$ for $\phi \in \operatorname{CProj}(J, g)$. 
Proposition 4.1. Suppose $(M, J, g)$ is a Kähler manifold of dimension $2 n \geq 4$ with degree of mobility 2 (i.e. $\operatorname{dim}(\mathcal{S})=2$ ). Let $\phi \in \operatorname{CProj}(J, g) \backslash \operatorname{Aff}(J, g)$ such that $\operatorname{det}\left(T_{\phi}\right)>0$. Then $T_{\phi} \in \mathrm{GL}(\mathcal{S}, \mathbb{R})$ has two distinct positive real eigenvalues.

Proof. Let $\phi \in \operatorname{CProj}(J, g) \backslash \operatorname{Aff}(J, g)$ such that $\operatorname{det}\left(T_{\phi}\right)>0$. Denote by $\mathcal{C}_{+}$the subset of elements in the 2-dimensional vector space $\mathcal{S}$ that are positive-definite at any point of $M$ and write $\eta \in \mathcal{C}_{+}$for the element in $\mathcal{S}$ corresponding to $g$. Evidently, $\mathcal{C}_{+}$forms a positive cone in $\mathcal{S}$, which is preserved by $T_{\phi}$. Note that our assumptions also imply that $T_{\phi}(\eta)$ and $\eta$ are linearly independent elements lying in $\mathcal{C}_{+}$(in particular, $T_{\phi}$ is not a constant multiple of the identity), since otherwise $\phi$ would necessarily be a homothety of $g$ and hence affine. This moreover implies that the cone $\mathcal{C}_{+}$has non-empty interior. Now set $\mathcal{C}:=\mathcal{C}_{+} \cup \mathcal{C}_{-}$, where $\mathcal{C}_{-}:=-\mathcal{C}_{+}$denotes the cone of elements in $\mathcal{S}$ that are negative definite at any point of $M$. We claim that the closure $\overline{\mathcal{C}}=\overline{\mathcal{C}}_{+} \cup \overline{\mathcal{C}}_{-}$of $\mathcal{C}$ does not coincide with $\mathcal{S}$, which together with the fact that $\mathcal{C}$ has non-empty interior implies that the boundary of $\mathcal{C}$ is the union of two distinct lines. Indeed, taking an appropriate linear combination of $\eta$ and $T_{\phi}(\eta)$, one can construct an element $\tilde{\eta} \in \mathcal{S}$ that at some point of $M$ is indefinite. Note that such an element $\tilde{\eta}$ can not be the limit of a sequence in $\mathcal{C}_{+}$or $\mathcal{C}_{-}$, that is, $\tilde{\eta}$ can neither be in $\overline{\mathcal{C}}_{+}$nor in $\overline{\mathcal{C}}_{-}$, which proves the claim. Since $T_{\phi}$ preserve the cone $\mathcal{C}_{+}$, it also preserves its boundary, which we have seen consists of two rays generated by two linearly independent elements in $\mathcal{S}$. The assumption $\operatorname{det}\left(T_{\phi}\right)>0$ in addition implies that the two rays of the boundary of $\mathcal{C}_{+}$are preserved individually, which shows that $T_{\phi}$ is diagonalisable with positive eigenvalues. Since, as already observed, $T_{\phi}$ is not a constant multiple of the identity, the claim follows.

Lemma 4.2. Suppose $(M, J, g)$ is a Kähler manifold of dimension $2 n \geq 4$ with degree of mobility 2 and denote by $\nabla$ the Levi-Civita connection of $g$. If there exists $\tilde{\eta} \in \mathcal{S}$ which is not parallel for $\nabla$, then any complex affine transformation is a homothety of $g$. In particular, if there exists $\phi \in \operatorname{CProj}(J, g) \backslash \operatorname{Aff}(J, g)$, then any complex affine transformation is a homothety of $g$.

Proof. Let $\eta$ denote the element in $\mathcal{S}$ corresponding to $g$ and assume $\phi$ is an element of $\operatorname{Aff}(J, g)$. Since $\operatorname{dim}(\mathcal{S})=2$ and $\{\eta, \tilde{\eta}\}$ is a basis of $\mathcal{S}$, we must have $\phi^{*} \eta=c \eta+d \tilde{\eta}$ for some constant $c, d \in \mathbb{R}$. Differentiating with respect to $\nabla$ yields

$$
0=\nabla \phi^{*} \eta=c \nabla \eta+d \nabla \tilde{\eta}=d \nabla \tilde{\eta}
$$

which implies $d=0$, since $\nabla \tilde{\eta} \neq 0$ by assumption.

Proposition 4.3. Suppose $(M, J, g)$ is a connected complete Kähler manifold of dimension $2 n \geq 4$ with degree of mobility 2 and assume that $\operatorname{Aff}(J, g) \subsetneq \operatorname{CProj}(J, g)$. Then for any $\phi \in \operatorname{CProj}(J, g) \backslash \operatorname{Aff}(J, g)$ we must have $\operatorname{det}\left(T_{\phi}\right)<0$. In particular, we have

$$
\phi, \psi \in \operatorname{CProj}(J, g) \backslash \operatorname{Aff}(J, g) \Longrightarrow \phi \circ \psi \in \operatorname{Aff}(J, g),
$$

i.e. the index of $\operatorname{Aff}(J, g)$ in $\operatorname{CProj}(J, g)$ is two.

Note that Proposition 4.3 shows that Theorem 1.2 holds under the assumption of degree of mobility 2, which is what remains to be shown to establish Theorem 1.2 .

4.2. Proof of Proposition 4.3. Throughout this section we suppose that:

- $(M, J, g)$ is a connected complete Kähler manifold of dimension $2 n \geq 4$ with LeviCivita connection $\nabla$,

- $\operatorname{dim}(\mathcal{S})=2$, 
- there exists $\phi \in \operatorname{CProj}(J, g) \backslash \operatorname{Aff}(J, g)$ such that $\operatorname{det}\left(T_{\phi}\right)>0$.

Our goal is to show that these assumptions lead to a contradiction, which proves Proposition 4.3 .

By Proposition 4.1, we know that the linear isomorphism $T_{\phi}: \mathcal{S} \rightarrow \mathcal{S}$ has two distinct positive real eigenvalues, which we denote by $\alpha>\beta>0$. Suppose $\eta, \tilde{\eta} \in \mathcal{S}$ are eigenvectors of $T_{\phi}$ corresponding to $\alpha$ respectively $\beta$. Since $\phi$ is by assumption not affine, the element $g^{-1} \operatorname{vol}(g)^{\frac{1}{n+1}} \in \mathcal{S}$ corresponding to the metric $g$ must be a linear combination $g^{-1} \operatorname{vol}(g)^{\frac{1}{n+1}}=c \eta+d \tilde{\eta}$ with $c, d \neq 0$. Hence, by rescaling the eigenvectors $\eta$ and $\tilde{\eta}$ if necessary, we may assume that

$$
g^{-1} \operatorname{vol}(g)^{\frac{1}{n+1}}=\eta+\tilde{\eta} .
$$

We also set $D_{a}^{b}=\eta^{c b} g_{a c} \operatorname{vol}(g)^{-\frac{1}{n+1}}$ and $\widetilde{D}_{a}^{b}=\tilde{\eta}^{c b} g_{a c} \operatorname{vol}(g)^{-\frac{1}{n+1}}$.

Note first that, since $(M, J, g)$ has degree of mobility 2 , the property of a point in $M$ to be regular with respect to an element $B \in \operatorname{Sol}(J, g)$, as defined in Definition 3.1, is invariant under c-projective transformations: If $B$ is a constant multiple of the identity, then any point is regular and so there is nothing to show. Assume now $B$ is not a constant multiple of Id and $x \in M$ a regular point with respect to $B$. Then $x$ is also regular with respect to any other element in $\operatorname{Sol}(J, g)$, since any element in $\operatorname{Sol}(J, g)$ is a linear combination of Id and $B$. For any $\psi \in \operatorname{CProj}(J, g)$ one has $\psi^{*} B \in \operatorname{Sol}\left(J, \psi^{*} g\right)$, which implies that $x$ is regular with respect to the endomorphism $\psi^{*} B$, since $\operatorname{Sol}\left(J, \psi^{*} g\right) \cong \operatorname{Sol}(J, g)$. Since the eigenvalues of $\psi^{*} B \in \operatorname{Sol}\left(J, \psi^{*} g\right)$ at $x$ coincide with the eigenvalues of $B$ at $\psi(x)$, we deduce that $\psi(x)$ is also regular with respect to $B$.

Let us now fix a regular point $x_{0} \in M$ with respect to a (hence any) $B \in \operatorname{Sol}(J, g)$ linearly independent to Id. Then there exists a neighbourhood $U$ of $x_{0}$ inside the set of regular points and, since $g$ is positive definite, a frame of $T U$, such that $g$ corresponds to the identity matrix and $D$ and $\tilde{D}$ to diagonal matrices $D=\operatorname{diag}\left(d_{1}, d_{1}, \ldots, d_{n}, d_{n}\right)$ respectively $\widetilde{D}=\operatorname{diag}\left(\tilde{d}_{1}, \tilde{d}_{1}, \ldots, \tilde{d}_{n}, \tilde{d}_{n}\right)$, where $d_{i}, \tilde{d}_{i}$ are smooth real-valued functions on $U$ with $d_{i}+\tilde{d}_{i}=$ 1 for $i=1, \ldots, n$. This implies that in the local frame the tensor

$$
A(k)_{a}^{b}:=\left(\phi^{-k}\right)^{*}\left(\eta^{b c}+\tilde{\eta}^{b c}\right) g_{a c} \operatorname{vol}(g)^{-\frac{1}{n+1}}
$$

corresponds to the following diagonal matrix:

$$
A(k)=\operatorname{diag}\left(\alpha^{k} d_{1}+\beta^{k} \tilde{d}_{1}, \alpha^{k} d_{1}+\beta^{k} \tilde{d}_{1}, \ldots, \alpha^{k} d_{n}+\beta^{k} \tilde{d}_{n}, \alpha^{k} d_{n}+\beta^{k} \tilde{d}_{n}\right) .
$$

Since $g$ and $\left(\phi^{-k}\right)^{*} g$ are positive definite, all diagonal entries of (19) are positive for all $k \in \mathbb{Z}$. Hence, $d_{i}+\left(\frac{\beta}{\alpha}\right)^{k} \tilde{d}_{i}>0$ respectively $\left(\frac{\alpha}{\beta}\right)^{k} d_{i}+\tilde{d}_{i}>0$ for all $k$ and taking the limit $k \rightarrow \infty$ respectively $k \rightarrow-\infty$ shows that $d_{i}, \tilde{d}_{i} \geq 0$ for all $i=1, \ldots, n$. Since $d_{i}+\tilde{d}_{i}=1$, we conclude that

$$
0 \leq d_{i} \leq 1 \quad \text { and } \quad 0 \leq \tilde{d}_{i} \leq 1 \quad \text { for all } i=1, \ldots, n .
$$

Consider the pull-back $\left(\phi^{-k}\right)^{*}(D)$, which corresponds in the frame over $U$ to a block diagonal matrix whose $i$-th block is given by

$$
\frac{\alpha^{k} d_{i}}{\alpha^{k} d_{i}+\beta^{k}\left(1-d_{i}\right)} \operatorname{Id}_{2}
$$

Recall that the eigenvalues of $\left(\phi^{-k}\right)^{*}(D)$ at $x_{0}$ are the same as the eigenvalues of $D$ at $\phi^{-k}\left(x_{0}\right)$. Since $\phi^{-k}\left(x_{0}\right)$ is again regular and the multiplicity of a constant eigenvalue of 
$D$ is constant on the set of regular points by Proposition 3.2, we therefore conclude that the only possible constant eigenvalues of $D$ on $U$ are 0 and 1 . Since $d_{i}=0$ (respectively $d_{i}=1$ ) on $U$ implies $\tilde{d}_{i}=1$ (respectively $\tilde{d}_{i}=0$ ), we deduce that the only possible constant eigenvalues of $A(k)$ are $\alpha^{k}$ and $\beta^{k}$. Since $U$ consists of regular points the distinct eigenvalues of $A(1)$ on $U$ are smooth real-valued functions with constant algebraic multiplicity. We write $2 m$ respectively $2 \tilde{m}$ for the algebraic multiplicities of the constant eigenvalues $\alpha$ and $\beta$ on $U$. The number of distinct non-constant eigenvalues of $A(1)$ is then given by $n-m-\tilde{m}$. Recall also that the algebraic multiplicities $2 m$ and $2 \tilde{m}$ of the constant eigenvalues $\alpha$ and $\beta$ of $A(1)$ do not depend on the choice of regular point $x_{0}$ by Proposition 3.2

Lemma 4.4. At $x_{0}$ and hence at any regular point we must have

$$
\alpha^{(n-\tilde{m})} \leq \beta^{-(\tilde{m}+1)} \text { and } \alpha^{(m+1)} \geq \beta^{-(n-m)},
$$

which implies

$$
n-m-\tilde{m}=1 \quad \alpha^{m+1}=\beta^{-(\tilde{m}+1)} .
$$

In CEMN the analogue statement (see Lemma 7.7 and the following considerations there) was proved for connected complete Kähler manifold $(M, J, g)$ of degree of mobility 2 under the assumption of the existence of a flow of non-affine c-projective transformations. The proof persists however in our discrete setting. For completeness and later purposes we nevertheless give the proof here again.

Proof. Note that $\left(\phi^{-k}\right)^{*} g=g(G(k) \cdot, \cdot)$, where $G(k):=\operatorname{det}_{\mathbb{R}}(A(k))^{-\frac{1}{2}} A(k)^{-1}$. Without loss of generality we assume that the first $2 \ell:=2 n-2 m-2 \tilde{m}$ diagonal entries of $D$ are not constant (which is equivalent to assuming that $d_{i}\left(x_{0}\right) \neq 0,1$ for $i=1, \ldots, \ell$ ), the next $2 m$ elements are equal to 1 , and the remaining $2 \tilde{m}$ elements are zero on $U$. Then, we deduce from (19) that $G(k)$ on $U$ is a block diagonal matrix of block sizes $2 \ell \times 2 \ell, 2 m \times 2 m$ and $2 \tilde{m} \times 2 \tilde{m}$ respectively, where the three blocks are given by

$$
\begin{gathered}
\Psi(t)\left(\begin{array}{ccc}
\frac{1}{d_{1} \alpha^{k}+\left(1-d_{1}\right) \beta^{k}} \operatorname{Id}_{2} & & \\
& \ddots & \\
& & \frac{1}{d_{\ell} \alpha^{k}+\left(1-d_{\ell}\right) \beta^{k}} \operatorname{Id}_{2}
\end{array}\right), \\
\Psi(t) \alpha^{-k} \operatorname{Id}_{2 m}, \quad \text { respectively } \quad \Psi(t) \beta^{-k} \operatorname{Id}_{2 \tilde{m}},
\end{gathered}
$$

where

$$
\Psi(t):=\alpha^{-k m} \beta^{-\tilde{m} k} \prod_{i=1}^{\ell} \frac{1}{d_{i} \alpha^{k}+\left(1-d_{i}\right) \beta^{k}} .
$$

Write $\nu_{1}, \ldots, \nu_{\ell}, \nu$ and $\tilde{\nu}$ for the eigenvalues of these respective diagonal matrices. Note that their asymptotic behaviour for $k \rightarrow+\infty$ respectively for $k \rightarrow-\infty$ is as follows

$$
\begin{array}{llll}
k \rightarrow+\infty & \nu_{i}(k) \sim \frac{\alpha^{-(n-\tilde{m}+1) k} \beta^{-\tilde{m} k}}{d_{i} \prod d_{j}} & \nu(k) \sim \frac{\alpha^{-(n-\tilde{m}+1) k} \beta^{-\tilde{m} k}}{\prod d_{j}} & \tilde{\nu}(k) \sim \frac{\alpha^{-(n-\tilde{m}) k} \beta^{-(\tilde{m}+1) k}}{\prod d_{j}} \\
k \rightarrow-\infty & \nu_{i}(k) \sim \frac{\alpha^{-m k} \beta^{-(n-m+1) k}}{\left(1-d_{i}\right) \prod\left(1-d_{j}\right)} & \nu(k) \sim \frac{\alpha^{-(m+1) k} \beta^{-(n-m) k}}{\prod\left(1-d_{j}\right)} & \tilde{\nu}(k) \sim \frac{\alpha^{-m k} \beta^{-(n-m+1) k}}{\prod\left(1-d_{j}\right)} .
\end{array}
$$

Assume now that (21) is not satisfied. Without loss of generality we can assume the first inequality is not satisfied, that is, we assume that $\alpha^{(n-\tilde{m})}>\beta^{-(\tilde{m}+1)}$, since otherwise we replace $\phi$ by $\phi^{-1}$, which exchanges the role of the inequalities in (21). 
Now consider the sequence $\left(\phi^{-k}\left(x_{0}\right)\right)_{k \in \mathbb{Z}>0}$. Then $\alpha^{(n-\tilde{m})}>\beta^{-(\tilde{m}+1)}$ implies that all eigenvalues of $G(k)$ decay exponentially as $\bar{k} \rightarrow \infty$ by (24). Hence, we conclude that the distance between $\phi^{-k}\left(x_{0}\right)$ and $\phi^{-(k+1)}\left(x_{0}\right)$ also decays at least exponentially as $k \rightarrow \infty$. This shows that $\left(\phi^{-k}\left(x_{0}\right)\right)_{k \in \mathbb{Z}_{>} 0}$ is a Cauchy sequence and hence completeness of $(M, J, g)$ implies that it converges. We denote the limit of $\left(\phi^{-k}\left(x_{0}\right)\right)_{k \in \mathbb{Z}_{>} 0}$ by $\tilde{x}$.

Now let $F$ be the smooth real-valued function on $M$ given by

$$
F=W_{a b}{ }_{d}^{c} W_{e f}{ }^{s} g_{c s} g^{a e} g^{b f} g^{d t},
$$

where $W_{a b}{ }^{c} d$ denotes the c-projective Weyl curvature of $(M, J,[\nabla])$ defined as in (9). Since $W_{a b}^{c}{ }_{d}$ is c-projectively invariant, $\left(\phi^{-k}\right)^{*} F\left(x_{0}\right)=F\left(\phi^{-k}\left(x_{0}\right)\right)$ equals

$$
\left.F\left(\phi^{-k}\left(x_{0}\right)\right)=\left(W_{a b}^{c}{ }_{d} W_{e f}^{s} t\right)\left(\phi^{-k}\right)^{*} g_{c s}\left(\phi^{-k}\right)^{*} g^{a e}\left(\phi^{-k}\right)^{*} g^{b f}\left(\phi^{-k}\right)^{*} g^{d t}\right)\left(x_{0}\right) .
$$

Since $F$ is continuous, we have $\lim _{k \rightarrow \infty} F\left(\phi^{-k}\left(x_{0}\right)\right)=F(\tilde{x})$.

In the frame we are working, the matrices corresponding to $g$ and $G(k)$ are diagonal and hence the function $F\left(\phi^{-k}\left(x_{0}\right)\right)$ is a sum of the form

$$
\sum_{1 \leq i, j, p, q \leq 2 n} C(i j p q ; k)\left(W_{i j}^{p} q\left(x_{0}\right)\right)^{2},
$$

where the coefficient $C(i j p q ; k)$ is the product of the $p$-th diagonal entry and the reciprocals of the $i$-th, $j$-th and $q$-th diagonal entry of the diagonal matrix that corresponds to $G(k)$ (and $W_{i j}{ }_{q}\left(x_{0}\right)$ denotes the coefficients of $W$ with respect to the frame). The coefficients $C(i j p q ; k)$ depend on $k$ and their asymptotic behaviour for $k \rightarrow \pm \infty$ can be read off from (24). Note moreover that all coefficients $C(i j p q ; k)$ are positive.

We claim that, if at least one of the indices $i, j$ or $q$ is less or equal than $2 n-2 m-2 \tilde{m}$, then $W_{i j}{ }^{p}\left(x_{0}\right)$ vanishes. Indeed, from (24) we conclude that $\left(\phi^{-k}\right)^{*} g$ decays exponentially at least as $\alpha^{-(n-\tilde{m}+1)} \beta^{-\tilde{m} k}$, which is up to a constant the smallest eigenvalue of $G(k)$, and that $\left(\phi^{-k}\right)^{*} g^{-1}$ goes exponentially to infinity at least as $\alpha^{(n-\tilde{m}) k} \beta^{(\tilde{m}+1) k}$ as $k \rightarrow \infty$. Suppose now that at least one of the indices $i, j$ or $q$ is less or equal than $2 n-2 m-2 \tilde{m}$. Then we deduce that up to multiplication by a positive constant $C(i j p q ; k)$ behaves asymptotically as $k \rightarrow \infty$ at least as

$$
\alpha^{(n-\tilde{m}) k} \beta^{(\tilde{m}+1) k} \alpha^{(n-\tilde{m}) k} \beta^{(\tilde{m}+1) k} \alpha^{(n-\tilde{m}+1) k} \beta^{\tilde{m} k} \alpha^{-(n-\tilde{m}+1) k} \beta^{-\tilde{m} k}=\alpha^{2(n-\tilde{m}) k} \beta^{2(\tilde{m}+1) k} .
$$

Since by assumption $\alpha^{(n-\tilde{m})}>\beta^{-(\tilde{m}+1)}$, we therefore conclude that the coefficient

$$
C(i j p q ; k) \rightarrow \infty \quad \text { as } k \rightarrow \infty .
$$

Since all terms in the sum (26) are nonnegative and the sequence $F\left(\phi^{-k}\left(x_{0}\right)\right)$ converges, we therefore deduce that $W_{i j}{ }^{p}\left(x_{0}\right)=0$ provided that at least one of the indices $i, j$ or $q$ is less or equal than $2 n-2 m-2 \tilde{m}$. Hence, there exist a non-zero vector $V \in T_{x_{0}} M$ such that

$$
W_{a b}{ }^{c} V^{a}=0, \quad W_{a b}{ }^{c}{ }_{d} V^{b}=0 \quad \text { and } \quad W_{a b}{ }^{c}{ }_{d} V^{d}=0,
$$

which shows that (3) of Remark 6.3 of [CEMN is satisfied, which implies that $(M, J, g)$ has so-called nullity at $x_{0}$ and hence on the set of regular points, since $x_{0}$ was arbitrary. Now Theorem 7.2 of [CEMN] says that, if $(M, J, g)$ is a connected complete Kähler manifold with nullity on a dense open set and whose holomorphic sectional curvature is not a positive constant (which is implied by our assumption that the degree of mobility is 2), then any complete Kähler metric that is c-projectively equivalent to $g$ is actually affinely equivalent to $g$, which contradicts our assumption that $\phi$ is not affine. Hence, the inequalities (21) must be satisfied. Now dividing the first inequality by the second shows $\alpha^{n-\tilde{m}-m-1} \leq \beta^{n-m-\tilde{m}-1}$, 
which implies that $n-\tilde{m}-m=1$, since $\alpha>\beta>0$ by assumption. Hence, $n-\tilde{m}=m+1$ and inserting this back into the (21) shows that $\alpha^{m+1}=\beta^{-(\tilde{m}+1)}$ as claimed.

To prove Proposition 4.3 it remains to show that also (22) leads to a contradiction. Here, we can not proceed as in CEMN, where the analogue statement was ruled out under the assumption of the existence a flow of non-affine c-projective transformations. Our strategy will be instead to show that under assumption (22) the Kähler manifold $(M, J, g)$ is necessarily compact. Then a similar reasoning as in the proof of Lemma 4.4 shows:

Lemma 4.5. If $M$ is compact, then (22) is not satisfied on the set of regular point of $M$.

Proof. Assume that the identities (22) are satisfied and fix a regular point $x_{0} \in M$. As in the proof of Lemma 4.4 consider the sequence $\left(\phi^{-k}\right)^{*} g=g(G(k) \cdot, \cdot)$. Note that the identities (22) now imply that the asymptotic behaviour (24) of the eigenvalues of $G(k)$ reads as follows:

$$
\begin{array}{cccc}
k \rightarrow+\infty & \nu_{1}(k) \sim \frac{1}{d_{1}^{2}}\left(\frac{\beta}{\alpha}\right)^{k} & \nu(k) \sim \frac{1}{d_{1}}\left(\frac{\beta}{\alpha}\right)^{k} & \tilde{\nu}(k) \sim \frac{1}{d_{1}} \\
k \rightarrow-\infty & \nu_{1}(k) \sim \frac{1}{\left(1-d_{1}\right)^{2}}\left(\frac{\alpha}{\beta}\right)^{k} & \nu(k) \sim \frac{1}{\left(1-d_{1}\right)} & \tilde{\nu}(k) \sim \frac{1}{\left(1-d_{1}\right)}\left(\frac{\alpha}{\beta}\right)^{k} .
\end{array}
$$

Now consider again $\left(\phi^{-k}\left(x_{0}\right)\right)_{k \in \mathbb{Z}>0}$. Note that in contrast to the reasoning in the proof of Lemma 4.4 the asymptotics (27) do not allow us to conclude that the sequence $\left(\phi^{-k}\left(x_{0}\right)\right)_{k \in \mathbb{Z}_{>}}$ is a Cauchy sequence (in fact there also counter examples). Since $M$ is compact, there is however a subsequence $\left(x_{\ell}\right)_{\ell \in \mathbb{Z}}:=\left(\phi^{-k_{\ell}}\left(x_{0}\right)\right)_{\ell \in \mathbb{Z}}$ of $\left(\phi^{-k}\left(x_{0}\right)\right)_{k \in \mathbb{Z}}$ that converges as $\ell \rightarrow \pm \infty$, where $k_{-\ell}=-k_{\ell}$. This implies that $F\left(x_{\ell}\right)$, which is given as in (26) by

$$
\sum_{1 \leq i, j, p, q \leq 2 n} C\left(i j p q ; k_{\ell}\right)\left(W_{i j}^{p}{ }_{q}\left(x_{0}\right)\right)^{2}
$$

converges as $\ell \rightarrow \pm \infty$. The coefficient $C\left(i j p q ; k_{\ell}\right)$ is the product of the $p$-th and the reciprocals of the $i$-th, $j$-th and $q$-th eigenvalues of $G\left(k_{\ell}\right)$ and from (27) we deduce that any such product either goes to $\infty$ or $-\infty$ as $\ell$ goes to $\infty$ respectively $-\infty$. Hence, $W_{a b}{ }^{c} d$ vanishes at $x_{0}$. Since $x_{0}$ was an arbitrary regular point, it vanishes on the open dense subset of regular points of $M$ and hence everywhere by connectedness of $M$. Hence, $(M, J, g)$ is c-projectively flat. Thus, the above mentioned Remark 6.3 and Theorem 7.2 of [CEMN] imply again that either $g$ has constant positive holomorphic sectional curvature or $\phi$ is affine, which contradict our assumptions.

Hence, if we can show that $M$ is compact, then Lemma 4.4 and Lemma 4.5 lead to a contradiction, which completes the proof of Proposition 4.3 .

In order to show that $M$ is compact, note that (22) implies that $D$ locally around any regular point (with respect to some adapted local frame) is of the form

$$
D=\left(\begin{array}{ccc}
\rho \mathrm{Id}_{2} & & \\
& \operatorname{Id}_{2 m} & \\
& & 0_{2 \tilde{m}}
\end{array}\right),
$$

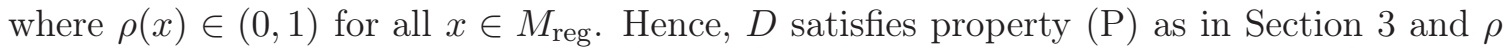
extends to a smooth function on all of $M$ with values in $[0,1]$. As in Section 3 we set again $M_{i}:=\{x \in M: \rho(x)=i\}$ for $i=0,1$ and denote by $\Lambda$ the gradient of $\rho$ with respect to $g$. 
Lemma 4.6. If (22) holds, then

$$
M_{i}:=\{x \in M: \rho(x)=i\} \neq \emptyset \quad \text { for } i=0,1,
$$

where $\rho=\frac{1}{2} D_{a}{ }^{a}-m$.

Proof. Let $\gamma: \mathbb{R} \rightarrow M$ be any arc-length parametrised geodesic such that $x_{0}:=\gamma(0) \in M_{\text {reg }}$ and $\gamma^{\prime}(0)$ is proportional to $\Lambda(\gamma(0))$ with a positive coefficient. Now let $I \subseteq \mathbb{R}$ with $0 \in I$ such that $\left.\gamma\right|_{I}$ satisfies the assumptions $(a-c)$ of Proposition 3.11. Since $\phi$ preserves the set of regular points, the point $\phi^{-1}(\gamma(0))$ is again a regular point. Hence, Proposition 3.11 implies that there exists a unique $s_{1} \in I^{o}$ such that $\phi^{-1}\left(x_{0}\right) \in L\left(\gamma\left(s_{1}\right)\right)$. Iterating this procedure, we obtain a sequence $\left(s_{k}\right)_{k \in \mathbb{Z}}$, where $s_{0}:=0$ and $s_{k} \in I^{o}$ is inductively defined by $\phi\left(\gamma\left(s_{k}\right)\right) \in L\left(\gamma\left(s_{k+1}\right)\right)$. By construction, we have $\rho\left(\gamma\left(s_{k}\right)\right)=\rho\left(\phi^{-k}\left(x_{0}\right)\right)$. From (20) we conclude that

$$
\rho\left(\gamma\left(s_{k}\right)\right)=\rho\left(\phi^{-k}\left(x_{0}\right)\right)=\frac{\alpha^{k} \rho\left(x_{0}\right)}{\alpha^{k} \rho\left(x_{0}\right)+\beta^{k}\left(1-\rho\left(x_{0}\right)\right)} .
$$

Hence, (22) implies that $\rho\left(\gamma\left(s_{k}\right)\right)$ is strictly increasing in $k$ and $\lim _{k \rightarrow \infty} \rho\left(\gamma\left(s_{k}\right)\right)=1$ respectively $\lim _{k \rightarrow-\infty} \rho\left(\gamma\left(s_{k}\right)\right)=0$. By assumption we moreover have $g\left(\gamma^{\prime}(s), \Lambda(\gamma(s))\right)>0$ on $I^{o}$, which implies that $s \mapsto \rho(\gamma(s))$ is strictly increasing on $I$. Hence, $\left(s_{k}\right)_{k \in \mathbb{Z}} \in I^{o}$ must be also strictly increasing. From Proposition 3.11 and the asymptotics (27), the length of the segment $\gamma_{\left[s_{k}, s_{k+1}\right]}$, which equals $s_{k+1}-s_{k}$, behaves asymptotically (up to a constant) as $\frac{\beta^{k}}{\alpha^{k}}$, which converges to 0 for $k \rightarrow \infty$. Hence, the limits $s_{ \pm}:=\lim _{k \rightarrow \pm \infty} s_{k}$ exist and $\rho\left(\gamma\left(s_{+}\right)\right)=\lim _{k \rightarrow \infty} \rho\left(\gamma\left(s_{k}\right)\right)=1$ respectively $\rho\left(\gamma\left(s_{-}\right)\right)=\lim _{k \rightarrow-\infty} \rho\left(\gamma\left(s_{k}\right)\right)=0$ imply $M_{i} \neq \emptyset$ for $i=0,1$.

By Lemma 4.6 and Theorem 3.4, we deduce that $M$ is compact. By Lemma 4.4 this is however a contradiction. Therefore, Proposition 4.3 holds, and, as a consequence, Theorem 1.2 is proved.

\section{Proof of Theorem 1.3}

Suppose that $(M, J, g)$ is a complete connected Kähler manifold satisfying the assumptions of Theorem 1.3. Since $g$ is not of constant positive holomorphic sectional curvature and $\operatorname{Aff}(J, g) \subsetneq \operatorname{CProj}(J, g)$, the degree of mobility of $(M, J, g)$ must be two by Theorem 1.4 and the index of $\operatorname{Aff}(J, g)$ in $\operatorname{CProj}(J, g)$ must be two by Theorem 1.2. By Lemma 4.2 moreover, the group $\operatorname{Aff}(J, g)$ must equal the group of homotheties of $g$. Hence, Theorem 1.3 follows from the fact that on a locally non-flat connected complete Riemannian manifold the group of homotheties coincides with the isometry group of $g$ [IO, Lemma 2].

\section{REFERENCES}

[ACG] V. Apostolov, D.M.J. Calderbank, P. Gauduchon: Hamiltonian 2-forms in Kähler geometry I: General theory, J. Diff. Geom. 73 (2006), 359-412.

[BMR] A.V. Bolsinov, V. S. Matveev, S. Rosemann: Local normal forms for c-projectively equivalent metrics and proof of the Yano-Obata conjecture in arbitrary signature. Proof of the projective Lichnerowicz conjecture for Lorentzian metrics; preprint arXiv:1510.00275.

[CMR] D.M.J. Calderbank, V.S. Matveev, S. Rosemann: Curvature and the c-projective mobility of Kähler metrics with hamiltonian 2-forms, Compositio Math. 152 (2016), 1555-1575.

[CEMN] D.M.J. Calderbank, M.G. Eastwood, V.S. Matveev, K. Neusser: C-projective geometry, to appear in Memoirs of AMS; preprint arXiv:1512.04516.

$[\mathrm{DM}]$ V.V. Domashev, J. Mikeš: On the theory of holomorphically projective mappings of Kählerian spaces, Math. Notes 23 (1978), 160-163; translation from Mat. Zametki 23 (1978), 297-304. 
[FKMR] A. Fedorova, V. Kiosak, V.S. Matveev, S. Rosemann: The only closed Kähler manifold with degree of mobility $\geq 3$ is $\left(C P(n), g_{\text {Fubini--Study }}\right)$, Proc. Lond. Math. Soc. 105 (2012), $153-188$.

[Fer1] J.Ferrand: Transformations conformes et quasi-conformes des variétés riemanniennes compactes, Acad. Roy. Bel. Cl. Sci. Mém. Coll. 39 (5)(1971),1-44.

[Fer2] J.Ferrand: The action of conformal transformations on a Riemannian manifold, Math. Ann. 304 (2)(1996), 277-291.

[F] C. Frances: Sur le group d'automorphismes des géométries paraboliques de rang un, Annales scientifiques de l'Ecole Normale Supérieure 40 (5)(2007), 741-764.

[KM] V. Kiosak, V.S.Matveev: Proof Of The Projective Lichnerowicz Conjecture For PseudoRiemannian Metrics With Degree Of Mobility Greater Than Two, Comm. Mat. Phys. 297 (2010), no.2, 401-426.

[IO] S.Ishihara, M.Obata: Affine transformations in a Riemannian manifold, Tohoku Math.J., Secon Series, 7 (3) (1955), 146-150.

[KT] K. Kiyohara, P.J. Topalov: On Liouville integrability of h-projectively equivalent Kähler metrics, Proc. Amer. Math. Soc. 139 (2011), 231-242.

[L] A. Lichnerowicz: Geometry of groups of transformations, translated from the French and edited by Michael Cole, Noordhoff International Publishing, Leyden (1977).

[M1] V.S. Matveev: Lichnerowicz-Obata conjecture in dimension two, Comm. Math. Helv. 81 (2005) no. 3, 541-570.

[M2] V.S. Matveev: Proof of projective Lichnerowicz-Obata conjecture, J. Diff. Geom. 75 (2007), 459-502.

[M3] V.S. Matveev: On the number of nontrivial projective transformations of closed manifolds (in Russian), Fundam. Prikl. Mat. 20 (2015), 125-131; an English translation was published in Journal of Math. Sciences 223 (2017), 734-738;

[M4] V.S. Matveev: Projectively invariant objects and the index of the group of affine transformations in the group of projective transformations, to appear in Bulletin of the Iranian Math. Soc, doi number 10.1007/s41980-018-0024-y; preprint arXiv:1604.01238.

[MR] V.S. Matveev, S. Rosemann: Proof of the Yano-Obata conjecture for h-projective transformations, J. Diff. Geom. 92 (2012), no. 2, 221-261, MR2998672.

[Mik] J. Mikes: Holomorphically projective mappings and their generalizations, Geometry, 3. J. Math. Sci. (New York) 89 (1998), no. 3, 1334-1353, MR1619720.

[Ob] M. Obata: The conjectures on conformal transformations of Riemannian manifolds, J. Diff. Geom. 6 (1971/72), 247-258.

[OT] T. Otsuki, Y. Tashiro: On curves in Kählerian spaces, Math. J. Okayama Univ. 4 (1954), $57-78$.

[Sch] R.Schoen: On the conformal and CR-automorphism groups, Geom.Funct.Anal. 5 (2)(1995), 464-481.

[S] N. S. Sinjukov: Geodesic mappings of Riemannian spaces, (in Russian) "Nauka", Moscow, 1979.

[Top] P. J. Topalov: Geodesic Compatibility And Integrability Of Geodesic Flows. Journal of Mathematical Physics 44 (2003), no. 2, 913-929.

[T] Y. Tashiro: On a holomorphically projective correspondence in an almost complex space, Math. J. Okayama Univ. 6 (1957), 147-152.

[Y] K. Yano: Differential geometry on complex and almost complex spaces. International Series of Monographs in Pure and Applied Mathematics, 49 A Pergamon Press Book. The Macmillan Co., New York 1965 xii +326 pp.

[Z] A. Zeghib: On discrete projective transformation groups of Riemannian manifolds, Adv. Math. 297 (2016), 26-53. 
Institut FÜr Mathematik, FAKUltät FÜr Mathematik Und Informatik, Friedrich-Schiller-Universität Jena, 07737 Jena, Germany

E-mail address: vladimir.matveev@uni-jena.de

Mathematical Institute, Faculty of Mathematics and Physics, Charles University, 18675 Prague, Czech Republic

E-mail address: kath.neusser@gmail.com 